\title{
Nicotine-mediated OTUD3 downregulation inhibits VEGF-C mRNA decay to promote lymphatic metastasis of human esophageal cancer
}

\section{Libing Song ( $\nabla$ songlb@sysucc.org.cn )}

Sun Yat-sen University Cancer Center, State Key Laboratory of Oncology in South China, Collaborative Innovation Center for Cancer Medicine

\section{Meng Wang}

State Key Laboratory of Oncology in South China, Collaborative Innovation Center for Cancer Medicine, Sun Yat-sen University Cancer Center

Yue Li

Sun Yat-sen University Cancer Center

\section{Yunyun Xiao}

State Key Laboratory of Oncology in South China, Collaborative Innovation Center for Cancer Medicine, Sun Yat-sen University Cancer Center

\section{Muwen Yang}

Sun Yat-sen University Cancer Center, State Key Laboratory of Oncology in South China, Collaborative Innovation Center for Cancer Medicine

\section{Yunting Jian}

Sun Yat-sen University Cancer Center, State Key Laboratory of Oncology in South China, Collaborative Innovation Center for Cancer Medicine

\section{Dongni Shi}

Sun Yat-sen University Cancer Center

\section{Xiangfu Chen}

State Key Laboratory of Oncology in South China, Collaborative Innovation Center for Cancer Medicine, Sun Yat-sen University Cancer Center

\section{Ying Ouyang}

State Key Laboratory of Oncology in South China, Collaborative Innovation Center for Cancer Medicine, Sun Yat-sen University Cancer Center

\section{Lingzhi Kong}

Sun Yat-sen University Cancer Center, State Key Laboratory of Oncology in South China, Collaborative Innovation Center for Cancer Medicine

\section{Xinjian Huang}

Sun Yat-sen University Cancer Center, State Key Laboratory of Oncology in South China, Collaborative Innovation Center for Cancer Medicine 


\section{Jiewen Bai}

Sun Yat-sen University Cancer Center, State Key Laboratory of Oncology in South China, Collaborative Innovation Center for Cancer Medicine

\section{Chuyong Lin}

Sun Yat-sen University Cancer Center

\section{Article}

Keywords: Esophageal cancer, nicotine, lymphatic metastasis, VEGF-C, mRNA decay, OTUD3

Posted Date: April 30th, 2021

DOl: https://doi.org/10.21203/rs.3.rs-379260/v1

License: (c) (i) This work is licensed under a Creative Commons Attribution 4.0 International License. Read Full License

Version of Record: A version of this preprint was published at Nature Communications on December 1st, 2021. See the published version at https://doi.org/10.1038/s41467-021-27348-8. 


\section{Nicotine-mediated OTUD3 downregulation inhibits VEGF-C mRNA decay to promote lymphatic metastasis of human esophageal cancer}

Short title: Nicotine inhibits VEGF-C mRNA decay

Meng Wang ${ }^{1, ~ \#, ~ Y u e ~ L i ~}{ }^{1}$, , Y unyun Xiao, \#, Muwen Yang ${ }^{1, ~ \#, ~ Y u n t i n g ~ J i a n ~}{ }^{1}$, Dongni Shi ${ }^{1}$, Xiangfu Chen ${ }^{1}$, Ying Ouyang ${ }^{1}$, Lingzhi Kong ${ }^{1}$, Xinjian Huang ${ }^{1}$, Jiewen Bai ${ }^{1}$, Chuyong $\operatorname{Lin}^{1,2, *}$, Libing Song ${ }^{1,3, *}$

${ }^{1}$ Department of Experimental Research, State Key Laboratory of Oncology in South China, Collaborative Innovation Center for Cancer Medicine, Sun Yat-sen University Cancer Center, Guangzhou 510060, China

${ }^{2}$ Guangdong Esophageal Cancer Institute, Guangzhou 510060, China

${ }^{3}$ Key Laboratory of Protein Modification and Degradation, School of Basic Medical Sciences; Affiliated Cancer Hospital \& Institute of Guangzhou Medical University, Guangzhou 511436, China

\# These authors contributed equally to this work.

* Corresponding author: Libing Song, Sun Yat-sen University Cancer Centre, Guangzhou, Guangdong 510060, China; Tel: +86(20)87343187; Fax: +86(20)87335828; E-mail: songlb@sysucc.org.cn.

Chuyong Lin, Sun Yat-sen University Cancer Centre, Guangzhou, Guangdong 510060, China; Tel: +86(20)87343187; Fax: +86(20)87335828; E-mail: linchy@sysucc.org.cn.

Conflict of interest: The authors declare no conflict of interest. 


\begin{abstract}
Nicotine addiction and the occurrence of lymph node spread are two major significant factors associated with esophageal cancer's poor prognosis; however, nicotine's role in inducing lymphatic metastasis of esophageal cancer remains unclear. Here we showed that OTU domain-containing protein 3 (OTUD3) was downregulated by nicotine and correlated with poor prognosis in heavy-smoking esophageal cancer patients. Nicotine-mediated OTUD3 downregulation promoted lymphatic metastasis by inducing substantial tumor-induced lymphangiogenesis. At the mechanistic level, OTUD3 directly interacted with ZFP36 ring finger protein (ZFP36) and stabilized it by inhibiting FBXW7-mediated K48-linked polyubiquitination. ZFP36 bound with the VEGF-C 3-'UTR and recruited the RNA degrading complex to induce its rapid mRNA decay. Thus, downregulation of OTUD3 and ZFP36 was essential for nicotine-induced VEGF-C production and lymphatic metastasis in esophageal cancer. This study establishes that the OTUD3/ZFP36/VEGF-C axis plays a vital role in nicotine addiction-induced lymphatic metastasis. It also suggests that OTUD3 may serve as a prognostic marker, and induction of the VEGF-C mRNA decay might be a potential therapeutic strategy against human esophageal cancer.
\end{abstract}

Keywords: Esophageal cancer; nicotine; lymphatic metastasis; VEGF-C; mRNA decay; OTUD3 


\section{Introduction}

Esophageal cancer ranks seventh in annual new incidence and sixth in mortality globally, with half of which occurs in China and a poor five-year survival rarely larger than $20 \%{ }^{1}$. Epidemiologic studies indicate that cigarette smoking is a significant prognostic factor, suggesting that smoking may further contribute to esophageal cancer's malignant progression ${ }^{2-4}$. On the other hand, the presence of tumor cells in regional or sentinel lymph nodes (LNs) is the most crucial feature of malignant esophageal cancer, directly inducing therapy resistance and tumor relapse ${ }^{5,6}$. Notably, retrospective studies showed that the smoking history is associated with LN metastasis status at the time of diagnosis and post-therapy in patients with esophageal ${ }^{7-10}$, oral ${ }^{11}$, lung ${ }^{12}$, and cutaneous ${ }^{13}$ cancers. Moreover, experimental data revealed that nicotine exposure promoted LN metastasis in head and neck squamous cell carcinoma ${ }^{14,15}$. However, the role of nicotine in inducing lymphatic metastasis of esophageal cancer remains unclear. Further research focusing on the mechanisms and key regulators in this process is necessary to design therapeutic strategies with the potential to control esophageal cancer progression.

Accumulating evidence indicates that cancer cells can enhance the development of lymphatic vessels within and near tumors, known as tumor-induced lymphangiogenesis, thereby robustly facilitating the LN dissemination of tumor cells ${ }^{16,17}$. Tumors provoke the growth and remodeling of lymphatic vessels by secreting pro-lymphangiogenic growth factors, such as the vascular endothelial growth factor C (VEGF-C). VEGF-C binds and activates the VEGFR3 signaling, promoting the proliferation, migration, and vascular permeability of lymphatic endothelial cells ${ }^{16-18}$. Consistently, VEGF-C is upregulated and promotes lymphatic metastasis in various human cancers, including esophageal ${ }^{19}$, bladder ${ }^{20}$, breast ${ }^{21}$, colorectal ${ }^{22}$, and lung cancer ${ }^{23}$. For example, we previously reported that transducin $(\beta)$-like $1 \mathrm{X}$-linked receptor 1 (TBL1XR1) increased VEGF-C transcription, thus promoted the lymphatic metastasis of esophageal cancer 19. Importantly, the VEGF-C blockage substantially reduced LN metastasis in mouse xenograft models ${ }^{19,20}$, suggesting that targeting VEGF-C may be a promising strategy. Unveiling the molecular mechanism underlying VEGF-C expression will hopefully open new avenues to prevent or overcome esophageal cancer.

Notably, recent studies reveal that decay of message RNA (mRNA) is emerging as a critical step for the expression of genes encoding cytokines ${ }^{24}$. Most of these transcripts are short-lived and contain conserved 
AU-rich elements (AREs) in their 3'-untranslated region (UTR). The AREs function as the cis-acting mediators and are recognized by trans-acting RNA-binding proteins such as ZFP36 ring finger protein (ZFP36/TTP) ${ }^{24-26}$. ZFP36 used its tandem CCCH zinc fingers to bind the ARE of substrate mRNAs. ZFP36 further recruits the mRNA decapping factor DCP2 ${ }^{27}$, CCR4-NOT deadenylase complex ${ }^{28}$, and RNA-degrading exosome ${ }^{29}$, thus mediating the rapid turnover of client mRNAs. For instance, ZFP36 has been identified as a direct suppressor of various cytokine mRNAs, including VEGFA, IL-6, IL-8, COX-2, IFN- $\gamma$, and TNF- $\alpha^{24}$. As a result, ZFP36 expression loss constitutively elevated cytokine productions, leading to sustained inflammation and malignant tumor progression ${ }^{24}$. Notably, a negative correlation between ZFP36 and VEGF-C was observed in uraemic rats with peritoneal dialysis ${ }^{30}$, indicating a potential regulation. Although VEGF-C transcription has been well-studied, how the mRNA decay of VEGF-C is inhibited in esophageal cancer to acquire high tumor-induced lymphangiogenesis capacity remains obscure.

Deubiquitinase (DUBs) are pleiotropic players regulating essentially every tumor hallmark by trimming or removing chains from attached proteins ${ }^{31}$. Approximately 100 human DUBs are known so far, some of which are identified as promising therapeutic targets ${ }^{31}$. Interestingly, we recently identified that OTU domain-containing protein 3 (OTUD3) was the most significantly changed DUB gene induced by the cigarette smoke extract (CSE). OTUD3 can deubiquitinate K48-linked ubiquitination to stabilize proteins ${ }^{32-34}$, and K63- or K11-linked ubiquitination to regulate protein activity ${ }^{35,36}$. The malfunction of OTUD3 facilitates the development and progression of human diseases, including cancers ${ }^{32-34}$. Notably, OTUD3 was downregulated in human cancers, contributing to enhanced cell proliferation, anti-apoptosis, and invasion ${ }^{32,33}$. However, the role of OTUD3 in smoking-mediated esophageal cancer progression remains unclear. This study found that nicotine-induced OTUD3 downregulation substantially promoted tumor-induced lymphangiogenesis and lymphatic metastasis in vivo, leading to poor clinical outcomes. We identified that OTUD3 inhibited the K48-linked ubiquitination and stabilized ZFP36, which further induced VEGF-C mRNA decay. Thus, ectopic expression of OTUD3 or ZFP36 abrogated nicotine-induced VEGF-C expression and lymphatic metastasis. These findings uncover a novel mechanism for nicotine addiction in facilitating lymphatic metastasis of esophageal cancer, and suggest that induction of VEGF-C mRNA decay is a potential therapeutic strategy. 


\section{Results}

\section{Nicotine-mediated OTUD3 downregulation correlates with lymph node metastasis and poor prognosis}

Deubiquitinases (DUBs) play critical roles in developing and progressing human cancers and might be targetable vulnerabilities ${ }^{31}$. Interestingly, our recent RNA-seq analysis showed that OTU domain-containing protein 3 (OTUD3) was the most significantly regulated DUB gene $\left(\left|\log _{2} \mathrm{Fc}\right|>1, \mathrm{P}<0.05\right)$ by cigarette smoke extract (CSE) in esophageal cancer cells (Figure 1A). Consistently, CSE treatment downregulated the OTUD3 mRNA expression in multiple esophageal cancer cell lines but not in the normal esophageal epithelial cell (NEEC) (Figure 1B). Notably, the main smoke substance nicotine and its derivatives dramatically reduced OTUD3 expression, while other tobacco carcinogens, such as Acetaldehyde and 4-Aminobiphenyl, had no significant effects on OTUD3 expression (Figure 1C). These findings reveal that nicotine could specifically downregulate OTUD3 in esophageal cancer cells.

PI3K/AKT, MAPK, and JAK2/STAT3 are the primary downstream signaling pathways of nicotine ${ }^{37}$. Interestingly, the PI3K inhibitor LY294002, but not the BRAF inhibitor or JAK2 inhibitor, abrogated nicotine-mediated OTUD3 downregulation (Supplementary Figure 1A-B). Indeed, the putative promoter of OTUD3 contained four potential binding sites of FOXO1 (Supplementary Figure 1C), suggesting that nicotine regulated OTUD3 expression through PI3K/AKT/FOXO1 signaling. As expected, promoter-reporter assays indicated that FOXO1 promoted OTUD3 transcription mainly via the third binding site (Supplementary Figure 1D). Notably, nicotine treatment significantly reduced the OTUD3 promoter activity and decreased the enrichment of FOXO1, p300, H3K27ac, and RNA polymerase II (pol II) on the OTUD3 promoter (Supplementary Figure 1E-F). These data reveal that nicotine downregulates OTUD3 via the PI3K/AKT/FOXO1 signaling in esophageal cancer.

We further assessed the clinical significance of OTUD3 expression in the 228 paraffin-embedded esophageal cancer specimens (Supplementary Table 1). Consistently, IHC staining revealed that OTUD3 was significantly downregulated in the heavy-smoking patients compared to those non-smokers, as well as those in the normal esophageal tissues (Figure 1D). Notably, LYVE-1 staining showed that the number of lymphatic vessels was robustly increased in esophageal tumors with low OTUD3 expression (Figure 1E). Moreover, correlation analysis indicated that the smoking history and low OTUD3 expression were 
significantly associated with LN metastasis of esophageal cancer (Figure 1F and Supplementary Table 2). Importantly, low expression of OTUD3 significantly predicted poorer tumor relapse-free survival (RFS) and overall survival (OS) in esophageal cancer patients (Figure $1 \mathrm{G}$ ), and this effect was more significant in the heavy-smoking patients than those non-smokers (Supplementary Figure 1G-H). Therefore, these results suggest that nicotine-mediated OTUD3 downregulation might contribute to lymphatic metastasis in the smoking esophageal cancer patients, leading to poor clinical outcomes.

\section{OTUD3 downregulation is required for nicotine-induced lymphatic metastasis}

Interestingly, direct nicotine administration in lymphatic endothelial cells (LECs) had no pro-lymphangiogenic effects (Supplementary Figure 2A-C), suggesting that nicotine might promote tumor-induced lymphangiogenesis (Supplementary Figure 2D). Strikingly, the conditioned medium (CM) derived from nicotine-treated esophageal cancer cells activated the VEGFR downstream signaling transduction in LECs (Figure 2A). Nicotine-induced CM promoted robust lymphangiogenesis as indicated by the increased migration and tube formation of LECs (Figure 2B-C). Importantly, restoration of OTUD3 abrogated nicotine-induced VEGFR signaling activation and lymphangiogenesis in vitro (Figure 2A-C). Likewise, the CM from OTUD3-silencing esophageal cancer cells significantly increased the migration and tube formation of LECs (Supplementary Figure 2D-G). These findings reveal that nicotine promotes tumor-induced lymphangiogenesis via the downregulation of OTUD3.

We further investigated the role of nicotine in lymphatic metastasis in vivo (Figure 2D). Briefly, mice were randomly divided into two groups ( $\mathrm{n}=12$ /group) and subcutaneously (s.c., daily) administered with vehicle or nicotine. Administration of nicotine in mice was validated by the serum levels of cotinine, the metabolite of nicotine (Supplementary Figure 2H). Each group was further equally divided and injected with control or OTUD3-overexpressing KYSE180 cells at the footpad. After a month of inoculation, mice were euthanized. The primary footpad tumors were subjected to lymphangiogenesis examination, while metastasis in lymph nodes was analyzed. Remarkably, nicotine promoted intra- and peri-tumoral lymphangiogenesis in vivo (Figure 2E). Moreover, administration of nicotine promoted LN metastasis as indicated by the volumes of LNs and the number of metastatic KYSE180 cells in LNs (Figure 2F-G). Additionally, the relative mRNA ratio of human HPRT1 to mouse GAPDH also indicated a higher proportion of colonized tumor cells in LNs 
${ }^{38}$ (Figure $\left.2 \mathrm{H}\right)$. Notably, ectopic expression of OTUD3 robustly prohibited the lymphangiogenesis and LN metastasis induced by nicotine (Figure 2E-H), suggesting that reduction of OTUD3 was indispensable for nicotine-induced lymphatic metastasis in esophageal cancer.

\section{Nicotine promotes VEGF-C expression through downregulation of OTUD3 and ZFP36}

VEGF-C and VEGF-D are the most well-established pro-lymphangiogenic factors ${ }^{16,17}$. Interestingly, we found that nicotine specifically increased the VEGF-C mRNA levels in a time-dependent manner but did not affect VEGF-D expression (Figure 3A and Supplementary Figure 3A). However, VEGF-C mRNA was retained at low levels and not affected by nicotine in OTUD3-overexpressing cells, indicating that repression of OTUD3 was requisite to VEGF-C upregulation (Figure 3A). Indeed, OTUD3 silencing robustly increased the mRNA and secreted protein levels of VEGF-C (Figure 3B and Supplementary Figure 3B). Notably, neither nicotine nor OTUD3 showed any significant effect on the VEGF-C promoter activity (Supplementary Figure 3C-D). These data suggested that the observed VEGF-C mRNA upregulation might not be caused via direct transcription but possibly in a post-transcriptional manner. As expected, the half-life of VEGF-C mRNA was prolonged in OTUD3-silencing cells but shortened in the OTUD3-overexpressing cells (Figure 3C), indicating that OTUD3 regulated VEGF-C mRNA decay.

The mRNA decay requires the involvement of RNA binding proteins (RBPs) ${ }^{26}$. Intriguingly, mass spectrometry analysis of OTUD3-interacting proteins concentrated on two RBPs, DDX3X and ZFP36 (Figure 3D and Supplementary Figure 3E). Of note, ZFP36 silencing, but not DDX3X, significantly increased VEGF-C mRNA expression (Figure 3E). In contrast, overexpression of ZFP36 reduced VEGF-C mRNA levels in a dose-dependent manner (Figure 3F). Notably, nicotine potently decreased ZFP36 expression in esophageal cancer cell lines and tumors, and these effects were abrogated by OTUD3 overexpression, suggesting that OTUD3 mediated the nicotine-induced ZFP36 downregulation (Figure 3G-H). These data reveal that ZFP36 may be the select RBP as downstream of OTUD3 to regulate VEGF-C mRNA decay .

OTUD3 did not regulate the mRNA expression of ZFP36 (Supplementary Figure 3F). Considering that OTUD3 can stabilize proteins, we speculated that OTUD3 might inhibit ZFP36 degradation. As expected, OTUD3 increased ZFP36 protein expression in a dose-dependent manner; however, the enzyme-dead 
OTUD3 ${ }^{\mathrm{C} 76 \mathrm{~A}}$ mutant had no effect (Figure 3I). Conversely, silencing of OTUD3 decreased ZFP36 in esophageal cancer cells, and this effect was abolished under the treatment of proteasome inhibitor MG132 (Figure 3J). Moreover, OTUD3 potently increased, while silencing of OTUD3 dramatically reduced the half-life of ZFP36 protein (Figure 3K). These findings indicate that OTUD3 stabilizes ZFP36 protein.

\section{OTUD3 inhibits the K48-linked ubiquitination of ZFP36}

We further investigated whether OTUD3 regulated the ubiquitination of ZFP36. Indeed, endogenous and exogenous reciprocal immunoprecipitation (IP) assays revealed that OTUD3 interacted with ZFP36 (Figure 4A-B). Notably, nicotine decreased the interactions between OTUD3 and ZFP36 as indicated by the IP and proximity ligation assays (PLAs) (Figure 4C and Supplementary Figure 4A). Further truncation IP assays revealed that the OTU domain of OTUD3 and N-terminal of ZFP36 were essential for their reciprocal interactions (Figure 4D). Moreover, in vitro binding assays using purified proteins showed that OTUD3 interacted with ZFP36 in a dose-dependent manner, suggesting that their interaction was direct (Figure 4E).

Remarkably, nicotine-mediated OTUD3 downregulation robustly increased the polyubiquitination of ZFP36, and this effect was abolished by OTUD3 re-expression (Figure 4F). Consistently, overexpression of OTUD3 diminished, while the silencing of OTUD3 promoted the polyubiquitination of ZFP36 (Figure 4G). Although OTUD3 ${ }^{\mathrm{C} 76 \mathrm{~A}}$ still interacted with ZFP36, it lost the ability to deubiquitinate ZFP36 (Figure 4G and Supplementary Figure 4B). Notably, OTUD3 specifically removed K48- but not K63-linked polyubiquitination of ZFP36 (Figure 4H and Supplementary Figure 4C).

The E3 ligase FBXW7 was recently identified to induce K48-linked polyubiquitination and degradation of ZFP36 ${ }^{39}$. Indeed, FBXW7 promoted the ubiquitination and degradation of ZFP36 in esophageal cancer cells (Figure 4I-J). Significantly, OTUD3 robustly impaired FBXW7-mediated ZFP36 ubiquitination and degradation in an enzyme-dependent manner (Figure 4I-J). These findings reveal that OTUD3 inhibits the ubiquitination and degradation of ZFP36.

\section{Nicotine inhibits VEGF-C mRNA decay}

We further explored the mechanism of VEGF-C mRNA decay. As expected, the VEGF-C 3'UTR contained a conserved AU-rich element (ARE, Figure 5A). Subsequently, RNA immunoprecipitation (RIP) assays revealed that ZFP36 bound explicitly with the VEGF-C, but not VEGF-D or ACTB mRNA (Figure 5B). 
Moreover, RNA pulldown assays indicated that ZFP36 interacted with the 3'UTR, but not 5'UTR, nor the CDS region of VEGF-C mRNA (Figure 5C). Mutation at ARE or competition using the non-biotin-labeled 3'UTR substantially diminished the interaction between ZFP36 and VEGF-C 3'UTR (Figure 5D-E). These observations indicate that ZFP36 interacts with the VEGF-C 3'UTR via ARE.

Once bound with substrate mRNA, ZFP36 recruits the CCR4-NOT deadenylase complex for subsequent mRNA degradation ${ }^{28}$. As expected, Our RIP assays indicated that the enrichment of ZFP36 and CNOT1 (CCR4-NOT deadenylase complex subunit 1) on VEGF-C mRNA was significantly reduced in ZFP36-silencing KYSE180 cells (Figure 5F). Consequently, ZFP36 overexpression induced rapid degradation of VEGF-C mRNA, while VEGF-C mRNA's half-life was prolonged by ZFP36 silencing (Figure 5G). Likewise, ZFP36 significantly reduced the luciferase activities conjugated with VEGF-C 3'UTR, but not those with ARE-mutant (Figure 5H). Moreover, ZFP36 regulated the half-life of luciferase mRNA conjugated with VEGF-C 3'UTR, but not the one with ARE-mutant (Supplementary Figure 5). These findings reveal that ZFP36 induces VEGF-C mRNA decay.

Notably, nicotine remarkably reduced the enrichment of ZFP36 on VEGF-C 3'UTR and increased the activity of luciferase-VEGF-C-3'UTR (Figure 5I-L). Thus, nicotine promoted the stability of VEGF-C mRNA (Figure 5M). Significantly, overexpression of OTUD3 or ZFP36 abrogated nicotine's effects on VEGF-C mRNA stabilization (Figure 5I-M). These findings suggest that nicotine inhibits VEGF-C mRNA decay via the downregulation of OTUD3 and ZFP36.

\section{Induction of VEGF-C mRNA decay abrogates lymphatic metastasis}

We further evaluated the potential of ZFP36-induced VEGF-C mRNA decay in lymphatic metastasis suppression. We overexpressed ZFP36 in nicotine-treated or OTUD3 silencing esophageal cancer cells (Supplementary Figure 6A). As expected, ZFP36 substantially reduced the VEGF-C mRNA expression in all these cell models, indicating that decay inhibition was essential for the robust VEGF-C production (Figure 6A). Consistently, ZFP36 overexpression potently inhibited tumor-induced lymphangiogenesis induced by nicotine or OTUD3 depletion, as indicated by LECs' migration and tube formation (Figure 6B-C). Strikingly, ZFP36 strongly reduced VEGF-C expression and the number of lymphatic vessels in the footpad tumors (Figure 6D-F). Consequently, ZFP36 abrogated the LN metastasis induced by nicotine or OTUD3 silencing 
(Figure 6G-I). These findings indicate that ZFP36 inhibits nicotine-induced lymphatic metastasis both in vitro and in vivo.

Notably, we previously found that TBL1XR1 transcriptionally upregulated VEGF-C to promote lymphangiogenesis ${ }^{19}$. We further tested whether ZFP36 overexpression blocked the role of TBL1XR1 (Supplementary Figure 6B). Significantly, ZFP36 strongly reduced VEGF-C mRNA levels in TBL1XR1-overexpressing esophageal cancer cells, resulting in robust inhibition of TBL1XR1-mediated LECs migration and tube formation (Supplementary Figure 6C-E). Taken together, these pieces of evidence suggest that induction of VEGF-C mRNA decay might be a promising therapeutic strategy against lymphatic metastasis in esophageal cancer.

\section{Clinical relevance and study model}

Finally, we assessed the OTUD3/ZFP36/VEGF-C axis's clinical relevance in esophageal cancer specimens. Like OTUD3, ZFP36 and VEGF-C expression correlated with smoking status in patients (Supplementary Figure 7). Significantly, IHC staining and correlation analysis showed that OTUD3 positively correlated with ZFP36 expression but was reversely associated with VEGF-C expression in the 228 esophageal cancer specimens (Figure 7A-B). Moreover, OTUD3 and ZFP36 were potently downregulated, while VEGF-C was elevated in the freshly collected esophageal cancer samples from the heavy-smoking patients compared to those non-smokers, suggesting a significant relevance of the OTUD3/ZFP36/VEGF-C axis in primary tumors (Figure 7C). Taken together, we here show that nicotine-mediated OTUD3 downregulation facilitates ZFP36 protein degradation and inhibits VEGF-C mRNA decay, leading to robust tumor-induced lymphangiogenesis and lymphatic metastasis in esophageal cancer. This study uncovers a novel mechanism for nicotine in lymphatic metastasis, indicating the great importance of smoking cessation, and suggesting that induction of VEGF-C mRNA decay might be a promising strategy (Figure 7D). 


\section{Discussion}

More than half of new cases and deaths of esophageal cancer occured in China ${ }^{1}$. The overall 5-year patient survival rate ranges from $15 \%$ to $25 \%$, mainly because patients are at an advanced stage at diagnosis, and the propensity for LN metastasis even when tumors are small ${ }^{1,40}$. The disseminated tumor cells in LNs are the direct cause of tumor relapse post-therapy ${ }^{5,6}$. Although a well-established causative relationship exists between smoking and several epithelial cancers ${ }^{41}$, the relation between smoking and the metastatic progression in esophageal cancer is not well studied. In this study, we found that smoking significantly correlated with LN metastasis status in clinical specimens. Moreover, using the mouse LN metastasis model, we demonstrated that nicotine exposure potently promoted esophageal cancer cells' lymphatic metastasis. These findings establish a promotive role of nicotine addiction in LN metastasis of esophageal cancer.

Notably, previous retrospective studies indicated that former smoking was related to the poor prognosis at diagnosis in several cancers, indicating that nicotine might even promote $\mathrm{LN}$ spread of tumor cells at the early stage. This hypothesis remains to be explored in the future, but it emphasizes the importance of smoking cessation among patients with or at risk for esophageal cancer. Of note, our data showed that low expression of OTUD3 had a much better prognostic value in heavy-smoking patients than the non-smokers.

This suggests that OTUD3 expression might be a potential prognostic marker for smoking patients.

The metastatic tropism of esophageal cancer in LNs is believed to be an active rather than passive process. Apart from migration and invasion, tumors can induce intra- and peri-tumoral lymphangiogenesis, providing numerous lymphatic vessels to facilitate metastasis ${ }^{16,17}$. Significantly, our previous and current studies showed that esophageal tumors that had higher indexes of intra- and peri-tumoral lymphatic vessels were much more prone to exhibit LN metastasis ${ }^{19}$. These data further strengthen the critical role of tumor-induced lymphangiogenesis in esophageal cancer progression. Notably, both studies identified that VEGF-C, rather than VEGF-D, was the more crucial pro-lymphangiogenic factor, suggesting that VEGF-C might be a promising therapeutic target in esophageal cancer.

Although the transcriptional regulation of VEGF-C is well-studied ${ }^{42}$, little is known about its post-transcriptional regulation. Notably, accumulating evidence reveals that balancing gene transcription and its mRNA decay is critical for cytokine productions ${ }^{24}$. The decay of mRNA is controlled by the cis-acting 
ARE sequence and trans-acting RBPs ${ }^{25,26}$. ZFP36 was identified as a suppressor of multiple inflammatory cytokines and was downregulated in many human cancers ${ }^{24}$. Notably, a reverse relation of ZFP36 and VEGF-C expression was found in uraemic rats with peritoneal dialysis; however, the underlying regulation remains unclear. In this study, we found that the VEGF-C mRNA had a conserved ARE in the 3'UTR. ZFP36 interacted with VEGF-C mRNA via the ARE and induced its rapid decay by recruiting the RNA degrading complex. Thus, lose of ZFP36 expression mediated nicotine-induced VEGF-C mRNA upregulation. Notably, ZFP36 abrogated the transcriptional or post-transcriptional upregulation of VEGF-C to retain it at a low level, resulting in substantial repression of lymphatic metastasis of esophageal cancer cells. Thus, these findings uncover a novel post-transcriptional mechanism for VEGF-C upregulation in cancer, and suggest that induction of mRNA decay by ZFP36 might be a promising strategy for VEGF-C blockage.

OTUD3 was reported to play a context-dependent role in cancers. It suppresses tumorigenesis in breast, colon, liver, brain, and cervical cancer by stabilizing PTEN and p53, while promoting lung tumorigenesis by stabilizing GRP78 ${ }^{32-34,43}$. Interestingly, our results indicated that OTUD3 was downregulated in primary esophageal tumors compared to normal esophageal tissues. OTUD3 expression was further repressed in smokers by nicotine via the PI3K/AKT/FOXO1 signaling. Moreover, low expression of OTUD3 was essential for nicotine-induced lymphatic metastasis and correlated with earlier tumor relapse and shorter patient survival. These observations further suggest that OTUD3 may play a tumor-suppressive role in esophageal cancer. Notably, OTUD3 plays pleiotropic functions by inducing different patterns of deubiquitination on various substrate proteins ${ }^{35,36}$. In this study, apart from ZFP36, we identified many other potential OTUD3-interacting proteins. It would be of great significance to further explore whether these proteins contribute to the tumor-suppressive role of OTUD3 in esophageal cancer.

In summary, our findings reveal that downregulation of OTUD3 and ZFP36 plays a critical role in nicotine-induced lymphatic metastasis in esophageal cancer by inhibiting the mRNA decay of VEGF-C. The study uncovers a novel mechanism for nicotine addiction in inducing lymphatic metastasis of esophageal cancer. It also suggests the great importance of smoking cessation and provides a novel therapeutic strategy against esophageal cancer. 


\section{Materials and Methods}

\section{Patient information and tissue specimens}

This study used 228 paraffin-embedded esophageal cancer patient specimens that had been clinically and histopathologically diagnosed at the Sun Yat-sen University Cancer Center from 2008 to 2015. The specimens were derived from 81 non-smoking patients who have never smoked before diagnosis, and 147 heavy-smoking patients defined by a pack-years (PY) smoking no less than 20 (PY $\geq 20$ ) as previously reported ${ }^{44}$. The clinicopathological characteristics are summarized in Supplementary Table 1. Freshly collected esophageal cancer tissues derived from 6 non-smokers and 6 heavy-smokers were frozen and stored in liquid nitrogen until use. Ethics approval was obtained from the Institutional Research Ethics Committee to use the clinical specimens for research purposes. Written informed consent was obtained from patients, and the study was carried out in accordance with the Declaration of Helsinki.

\section{Immunohistochemistry (IHC)}

IHC staining was performed on the 228 paraffin-embedded esophageal cancer tissue sections using anti-OTUD3 (Sigma-Aldrich, HPA028544), anti-LYVE-1 (Sigma-Aldrich, HPA042953), anti-ZFP36 (Merck Millipore, ABE285), and anti-VEGF-C (R\&D, AF752) antibodies as previously described ${ }^{19}$. The staining results were evaluated and scored by two independent pathologists, who were blinded to the clinical outcome. The staining was graded with four scores, strong +3 , moderate +2 , weak +1 , and negative 0 . Specimens with scores $+3,+2$ were defined as high expression, while the others scored as +1 or 0 were the low expression. The number of lymphatic vessels was measured by counting LYVE-1-positive vessels as previously reported

45. Under a magnification of 200X microscope, 10 fields of view were randomly selected for each slice, and the average number of LYVE-1-positive lymphatic vessels was counted and the whole number was kept.

Details of the IHC method were provided in the supplementary information.

\section{Inguinal lymph node metastasis model}

The inguinal lymph node metastasis model was performed as previously reported ${ }^{19}$. BALB/c-nude mice (Male, 4-5 weeks old) were purchased and housed in barrier facilities on a $12 \mathrm{~h}$ light/dark cycle. All experimental procedures were approved by the Institutional Animal Care and Use Committee of Sun Yat-sen University and performed following the Declaration of Helsinki. Briefly, mice were randomly divided into 
two groups ( $\mathrm{n}=12 /$ group$)$. Nicotine $(0.75 \mathrm{mg} / \mathrm{kg}$, s.c., daily) was administered with a $4 \mathrm{~mm}$ needle in mice to create a smoking-like microenvironment as previously reported ${ }^{46}$. Nicotine administration in mice was validated by examining the plasma cotinine (nicotine metabolite) levels using ELISA assay. Each group was further equally divided and injected with control or OTUD3-overexpressing KYSE180 cells $\left(5 \times 10^{5}\right)$ at the footpad. After a month of inoculation, mice were euthanized. The primary footpad tumors were paraffin-embedded and subjected to H\&E and IHC staining. Inguinal lymph nodes (LN) were first measured to calculate volumes. Parts of LNs were used for RNA extraction and qRT-PCR analysis of hHRPT1 and mGAPDH. The remaining LNs were then paraffin-embedded and subjected to IHC staining of squamous cell carcinoma marker p63.

\section{Ployubiquitination analysis}

To analyze the polyubiquitylation of ZFP36, we treated cells with $10 \mu \mathrm{M}$ of the proteasome inhibitor MG132 for $6 \mathrm{~h}$. The cells were washed with PBS, pelleted, and lysed in HEPES buffer (20 mM HEPES, pH 7.2, 50 $\mathrm{mM} \mathrm{NaCl}, 1 \mathrm{mM} \mathrm{NaF}, 0.5 \%$ Triton X-100) plus $0.1 \% \mathrm{SDS}, 10 \mu \mathrm{M}$ MG132 and protease-inhibitor cocktail. The lysates were centrifuged to obtain cytosolic proteins and incubated with anti-ZFP36 (Merck Millipore, ABE285) overnight. The lysates were then pulldown with agarose beads. The beads were washed six times with HEPES buffer and then eluted with $200 \mu \mathrm{l}$ of $1 \mathrm{M}$ glycine ( $\mathrm{pH}$ 3.0). The proteins were boiled in SDS-PAGE sample buffer for 5 min and analyzed by immunoblotting with an anti-Ub antibody (Cell Signaling Technology, \#3936).

\section{RNA IP (RIP) assays}

This study performed RIP assays to examine the interactions between ZFP36 and VEGF-C mRNA in esophageal cancer cells. Briefly, cells lysed in the lysis buffer (20mM Tris-Cl, $\mathrm{pH} 8.0,10 \mathrm{mM} \mathrm{NaCl}, 1 \mathrm{mM}$ EDTA, $0.5 \%$ NP-40) supplemented with RNasin (Promega). Lysates were then pulldown with anti-ZFP36 (Merck Millipore, ABE285) or anti-CNOT1 (Bethyl, A305-787A) antibody, washed five times with the lysis buffer. The retrieved pellets were then subjected to RT-PCR analysis using the qRT-PCR primers of VEGF-C-3'UTR. ACTB was used as a negative control. Primers were provided in the supplementary information.

\section{RNA synthesis, labeling, and pulldown}


RNA for in-vitro experiments was transcribed with T7 or SP6 RNA polymerase (Ambion) from PCR amplified templates with T7 forward 5'-TAATACGACTCACTATAG-3' and SP6 Reverse 5'-ATTTAGGTGACACTATAG-3' primers. According to the manufacturer's instructions, the RNA fragments, including 5'UTR, CDS, 3'UTR, 3'UTR-mut, and 3'UTR-AS, were purified and biotin-labeled using RNA 3'-End Biotinylation Kit (Thermo Fisher Scientific). The in vitro binding assays of biotin-labeled VEGF-C mRNA fragments and ZFP36 protein were performed. Briefly, cells $\left(1 \times 10^{7}\right)$ were lysated with $1 \mathrm{ml}$ of binding buffer (50 mM Tris-HCl pH 7.9, 10\% glycerol, $100 \mathrm{mM} \mathrm{KCl,} 5 \mathrm{mM} \mathrm{MgCl} 2,10 \mathrm{mM} \beta-\mathrm{ME}, 0.1 \%$ NP-40, 1 mM PMSF, 1 x Superase-in, and 1 x protease inhibitor cocktail). Labeled RNA (50 pmol) was incubated with cell lysates for $1 \mathrm{~h}$ at RT. Then, $50 \mu \mathrm{l}$ of washed streptavidin-conjugated magnetic beads (Thermo Fisher Scientific) was added to each reaction and incubated at RT for $30 \mathrm{~min}$. Beads were washed five times, and the retrieved protein was subjected to western blot analysis.

\section{RNA-seq data}

KYSE180 cells were treated with DMSO or CSE for $24 \mathrm{~h}$ and then subjected to RNA extraction and RNA-seq analysis. The RNA-seq data have been deposited in the National Center for Biotechnology Information Sequence Read Archive (SRA) database (https://www.ncbi.nlm.nih.gov/sra/PRJNA678868).

\section{Statistical analysis}

Statistical analyses were performed using the SPSS version 19.0 statistical software package. Statistical tests for data analysis included the log-rank test, $\chi^{2}$ test, and Student's t-test (two-tailed). Multivariate statistical analysis was performed using a Cox regression model. $P<0.05$ was considered statistically significant. *, $P$ $<0.05 ; * *, P<0.01 ; * * *, P<0.001$.

Additional information is provided in Supplementary Materials and Methods. 


\section{Author Contribution Statement}

M.W., Y.L., Y.X., and M.Y. carried out most of the experimental work; they collected and analyzed the data. M.W., Y.X., X.C., and X.H. conducted the western blot analysis, plasmid constructions, IP assays. Y.L., M.Y., Y.J., and D.S. conducted the RNA-seq, qRT-PCR, luciferase reporters, ChIP, and PLA assays. M.Y., Y.X., and L.K. collected tissues, patient information, and conducted IHC and survival analysis. M.W., Y.L, and X.C. conducted animal studies. Y.O. and J.B. conducted cell culture. M.Y. and Y.X. performed the in vitro studies. C.L. and L.S. raised the concept, design the experiments, wrote the manuscript, and supervised the project.

\section{Acknowledgments}

This work was supported by the National Key Research and Development Program of China (No. 2020YFA0509400), National Natural Science Foundation of China (Nos. 81672854, 81530082, 81872383, 81773106, 82072609, and 91740118), Natural Science Foundation of Guangdong Province (Nos. 2017A030306019, 2018B030311060), Pearl River S\&T Nova Program of Guangzhou (No.201710010163), the Fundamental Research Funds for the Central Universities (No. 19ykzd45), the Program of Guangdong Esophageal Cancer Institute (M201805), and the program for Excellent Talents in Cancer Centre (No. 16zxyc01). 


\section{References}

1 Bray, F. et al. Global cancer statistics 2018: GLOBOCAN estimates of incidence and mortality worldwide for 36 cancers in 185 countries. CA: a cancer journal for clinicians $\mathbf{6 8}, 394-424$, doi:10.3322/caac.21492 (2018).

2 Kuang, J. J. et al. Smoking Exposure and Survival of Patients with Esophagus Cancer: A Systematic Review and Meta-Analysis. Gastroenterology research and practice 2016, 7682387, doi:10.1155/2016/7682387 (2016).

3 McCain, R. S. et al. Alcohol intake, tobacco smoking, and esophageal adenocarcinoma survival: a molecular pathology epidemiology cohort study. Cancer causes \& control 31, 1-11, doi:10.1007/s10552-019-01247-2 (2020).

4 Cui, R. et al. Functional variants in ADH1B and ALDH2 coupled with alcohol and smoking synergistically enhance esophageal cancer risk. Gastroenterology 137, 1768-1775, doi:10.1053/j.gastro.2009.07.070 (2009).

5 Hosch, S. B. et al. Esophageal cancer: the mode of lymphatic tumor cell spread and its prognostic significance. Journal of clinical oncology 19, 1970-1975 (2001).

6 Leng, X. et al. Prognostic Impact of Postoperative Lymph Node Metastases After Neoadjuvant Chemoradiotherapy for Locally Advanced Squamous Cell Carcinoma of Esophagus: From the Results of NEOCRTEC5010, a Randomized Multicenter Study. Annals of surgery, doi:10.1097/SLA.0000000000003727 (2019).

$7 \quad$ Liu, L., Huang, C., Liao, W., Chen, S. \& Cai, S. Smoking behavior and smoking index as prognostic indicators for patients with esophageal squamous cell carcinoma who underwent surgery: A large cohort study in Guangzhou, China. Tob Induc Dis 18, 9, doi:10.18332/tid/117428 (2020).

8 Zheng, Y. et al. Smoking affects treatment outcome in patients with resected esophageal squamous cell carcinoma who received chemotherapy. PloS one 10, e0123246, doi:10.1371/journal.pone.0123246 (2015).

9 Zou, G. R., Su, Z., Li, J. Y., Xie, F. Y. \& Li, Q. Prognostic impact of cigarette smoking on the survival of patients with established esophageal squamous cell carcinoma receiving radiotherapy: A retrospective study from southern China. Exp Ther Med 17, 3671-3681, doi:10.3892/etm.2019.7356 (2019).

10 Abrams, J. A., Lee, P. C., Port, J. L., Altorki, N. K. \& Neugut, A. I. Cigarette smoking and risk of lung metastasis from esophageal cancer. Cancer epidemiology, biomarkers \& prevention 17, 2707-2713, doi:10.1158/1055-9965.EPI-08-0232 (2008).

11 Hussain, A. et al. Association of smoking with lymph node metastasis in early stages of squamous cell carcinoma of tongue. J Coll Physicians Surg Pak 15, 273-275, doi:05.2005/JCPSP.273275 (2005).

12 Ding, N. et al. Predictors of lymph node metastasis and possible selective lymph node dissection in clinical stage IA non-small cell lung cancer. Journal of thoracic disease 10, 4061-4068, doi:10.21037/jtd.2018.06.129 (2018).

13 Jones, M. S. et al. The Impact of Smoking on Sentinel Node Metastasis of Primary Cutaneous Melanoma. Annals of surgical oncology 24, 2089-2094, doi:10.1245/s10434-017-5775-9 (2017).

14 Shimizu, R. et al. Nicotine promotes lymph node metastasis and cetuximab resistance in head and neck squamous cell carcinoma. International journal of oncology 54, 283-294, doi:10.3892/ijo.2018.4631 (2019).

15 Wang, M. et al. Nicotine promotes cervical metastasis of oral cancer by regulating peroxiredoxin 1 and epithelial-mesenchymal transition in mice. OncoTargets and therapy 12, 3327-3338, doi:10.2147/OTT.S194129 (2019).

16 Stacker, S. A. et al. Lymphangiogenesis and lymphatic vessel remodelling in cancer. Nature reviews. Cancer 14, 159-172, doi:10.1038/nrc3677 (2014). 
17 Stacker, S. A., Achen, M. G., Jussila, L., Baldwin, M. E. \& Alitalo, K. Lymphangiogenesis and cancer metastasis. Nature reviews. Cancer 2, 573-583, doi:10.1038/nrc863 (2002).

Joukov, V. et al. A novel vascular endothelial growth factor, VEGF-C, is a ligand for the Flt4 (VEGFR-3) and KDR (VEGFR-2) receptor tyrosine kinases. The EMBO journal 15, 290-298 (1996).

Liu, L. et al. TBL1XR1 promotes lymphangiogenesis and lymphatic metastasis in esophageal squamous cell carcinoma. Gut 64, 26-36, doi:10.1136/gutjnl-2013-306388 (2015).

Miyata, Y. et al. Lymphangiogenesis and angiogenesis in bladder cancer: prognostic implications and regulation by vascular endothelial growth factors-A, -C, and -D. Clinical cancer research $\mathbf{1 2}$, 800-806, doi:10.1158/1078-0432.CCR-05-1284 (2006).

Skobe, M. et al. Induction of tumor lymphangiogenesis by VEGF-C promotes breast cancer metastasis. Nature medicine 7, 192-198, doi:10.1038/84643 (2001).

Tacconi, C. et al. Vascular endothelial growth factor $\mathrm{C}$ disrupts the endothelial lymphatic barrier to promote colorectal cancer invasion. Gastroenterology 148, 1438-1451 e1438, doi:10.1053/j.gastro.2015.03.005 (2015).

Renyi-Vamos, F. et al. Lymphangiogenesis correlates with lymph node metastasis, prognosis, and angiogenic phenotype in human non-small cell lung cancer. Clinical cancer research 11, 7344-7353, doi:10.1158/1078-0432.CCR-05-1077 (2005).

Guo, J., Qu, H., Chen, Y. \& Xia, J. The role of RNA-binding protein tristetraprolin in cancer and immunity. Medical oncology 34, 196, doi:10.1007/s12032-017-1055-6 (2017).

Chen, C. Y. \& Shyu, A. B. AU-rich elements: characterization and importance in mRNA degradation. Trends in biochemical sciences 20, 465-470, doi:10.1016/s0968-0004(00)89102-1 (1995).

Garcia-Maurino, S. M. et al. RNA Binding Protein Regulation and Cross-Talk in the Control of AU-rich mRNA Fate. Front Mol Biosci 4, 71, doi:10.3389/fmolb.2017.00071 (2017).

Fenger-Gron, M., Fillman, C., Norrild, B. \& Lykke-Andersen, J. Multiple processing body factors and the ARE binding protein TTP activate mRNA decapping. Molecular cell 20, 905-915, doi:10.1016/j.molcel.2005.10.031 (2005).

Fabian, M. R. et al. Structural basis for the recruitment of the human CCR4-NOT deadenylase complex by tristetraprolin. Nature structural \& molecular biology 20, 735-739, doi:10.1038/nsmb.2572 (2013).

Chen, C. Y. et al. AU binding proteins recruit the exosome to degrade ARE-containing mRNAs. Cell 107, 451-464, doi:10.1016/s0092-8674(01)00578-5 (2001).

30 Xiao, J. et al. The abnormal expressions of tristetraprolin and the VEGF family in uraemic rats with peritoneal dialysis. Molecular and cellular biochemistry 392, 229-238, doi:10.1007/s11010-014-2033-3 (2014).

Fraile, J. M., Quesada, V., Rodriguez, D., Freije, J. M. \& Lopez-Otin, C. Deubiquitinases in cancer: new functions and therapeutic options. Oncogene 31, 2373-2388, doi:10.1038/onc.2011.443 (2012).

32 Yuan, L. et al. Deubiquitylase OTUD3 regulates PTEN stability and suppresses tumorigenesis. Nature cell biology 17, 1169-1181, doi:10.1038/ncb3218 (2015).

Pu, Q., Lv, Y. R., Dong, K., Geng, W. W. \& Gao, H. D. Tumor suppressor OTUD3 induces growth inhibition and apoptosis by directly deubiquitinating and stabilizing p53 in invasive breast carcinoma cells. BMC cancer 20, 583, doi:10.1186/s12885-020-07069-9 (2020).

$\mathrm{Du}, \mathrm{T}$. et al. The deubiquitylase OTUD3 stabilizes GRP78 and promotes lung tumorigenesis. Nature communications 10, 2914, doi:10.1038/s41467-019-10824-7 (2019).

35 Zhang, Z. et al. Acetylation-Dependent Deubiquitinase OTUD3 Controls MAVS Activation in Innate Antiviral Immunity. Molecular cell 79, 304-319 e307, doi:10.1016/j.molcel.2020.06.020 (2020).

Flierman, D. et al. Non-hydrolyzable Diubiquitin Probes Reveal Linkage-Specific Reactivity of Deubiquitylating Enzymes Mediated by S2 Pockets. Cell Chem Biol 23, 472-482, 
doi:10.1016/j.chembiol.2016.03.009 (2016).

37 Grando, S. A. Connections of nicotine to cancer. Nature reviews. Cancer 14, 419-429, doi:10.1038/nrc3725 (2014).

$38 \mathrm{Yu}, \mathrm{F}$. et al. let-7 regulates self renewal and tumorigenicity of breast cancer cells. Cell 131, 1109-1123, doi:10.1016/j.cell.2007.10.054 (2007).

39 Zhang, Z. et al. RNA-binding protein ZFP36/TTP protects against ferroptosis by regulating autophagy signaling pathway in hepatic stellate cells. Autophagy 16, 1482-1505, doi:10.1080/15548627.2019.1687985 (2020).

40 Pennathur, A. et al. Esophagectomy for T1 esophageal cancer: outcomes in 100 patients and implications for endoscopic therapy. The Annals of thoracic surgery 87, 1048-1054; discussion 1054-1045, doi:10.1016/j.athoracsur.2008.12.060 (2009).

41 Chen, Z. M., Xu, Z., Collins, R., Li, W. X. \& Peto, R. Early health effects of the emerging tobacco epidemic in China. A 16-year prospective study. Jama 278, 1500-1504, doi:10.1001/jama.278.18.1500 (1997).

42 Chilov, D. et al. Genomic organization of human and mouse genes for vascular endothelial growth factor C. The Journal of biological chemistry 272, 25176-25183 (1997).

43 Liu, Y. Z., Du, X. X., Zhao, Q. Q., Jiao, Q. \& Jiang, H. The expression change of OTUD3-PTEN signaling axis in glioma cells. Annals of translational medicine 8, 490, doi:10.21037/atm.2020.03.51 (2020).

44 Shitara, K. et al. Heavy smoking history interacts with chemoradiotherapy for esophageal cancer prognosis: a retrospective study. Cancer science 101, 1001-1006, doi:10.1111/j.1349-7006.2009.01466.x (2010).

45 Bono, P. et al. High LYVE-1-positive lymphatic vessel numbers are associated with poor outcome in breast cancer. Clinical cancer research 10, 7144-7149, doi:10.1158/1078-0432.CCR-03-0826 (2004).

46 Lefever, T. W., Thomas, B. F., Kovach, A. L., Snyder, R. W. \& Wiley, J. L. Route of administration effects on nicotine discrimination in female and male mice. Drug Alcohol Depend 204, 107504, doi:10.1016/j.drugalcdep.2019.06.007 (2019). 
Figure 1

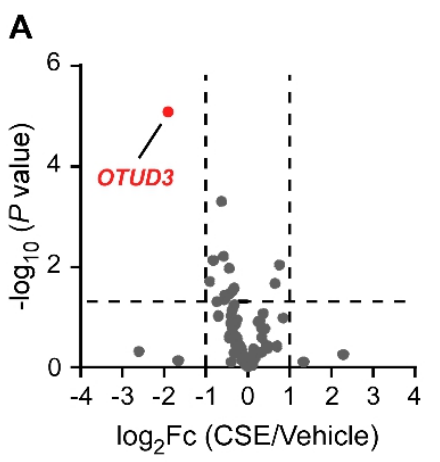

B

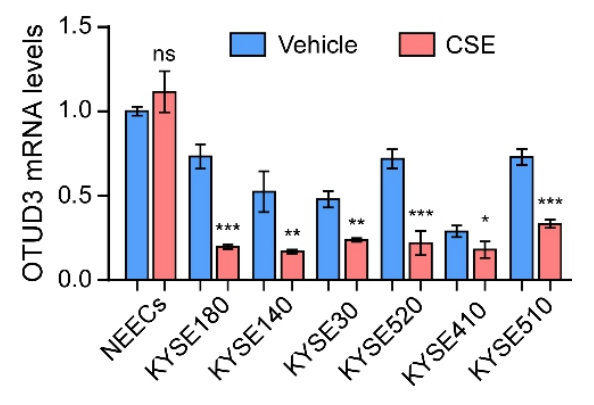

C

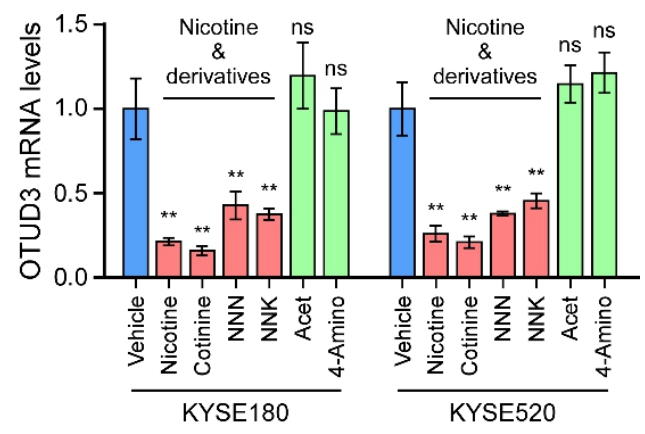

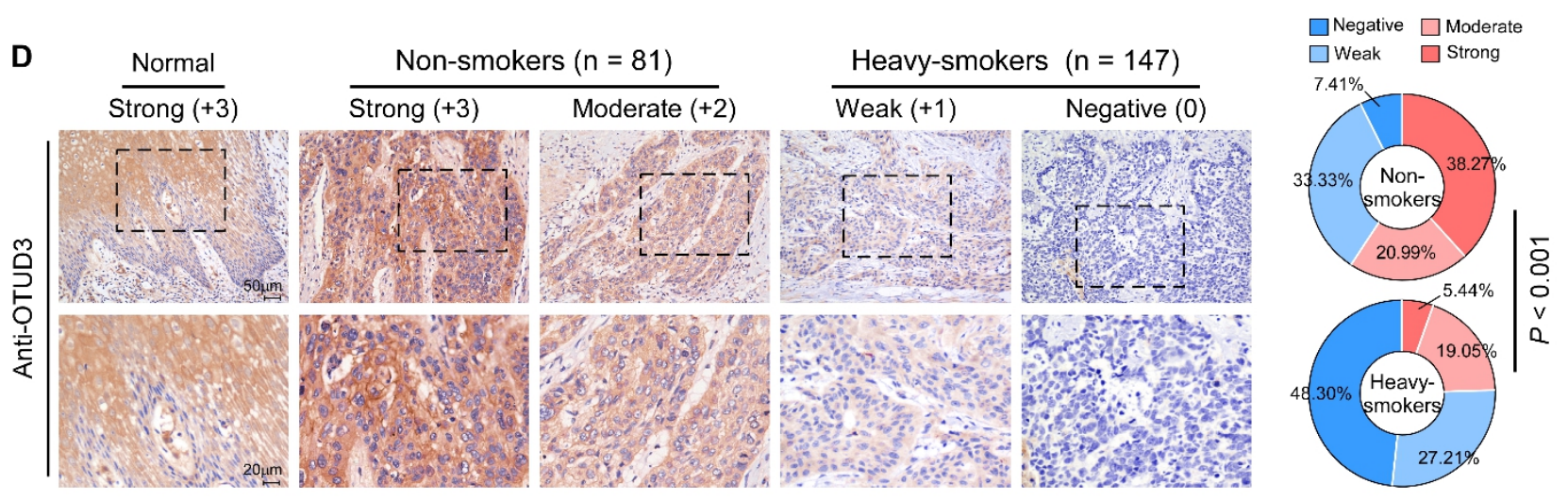

E
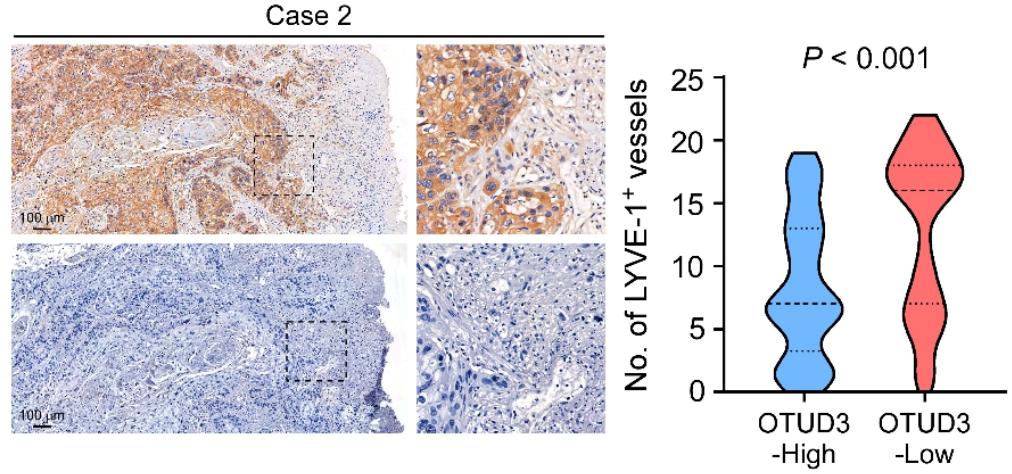

$\mathbf{F}$

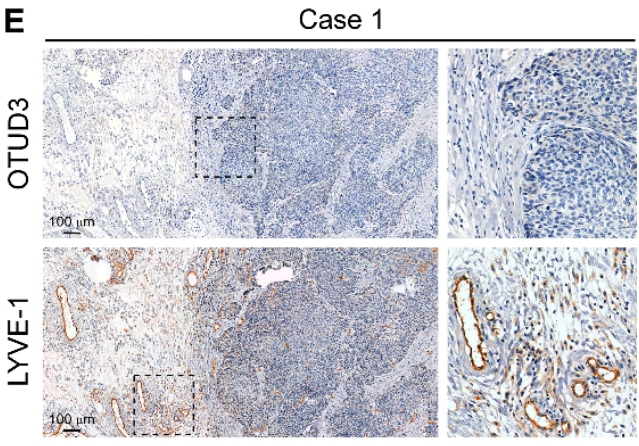

G
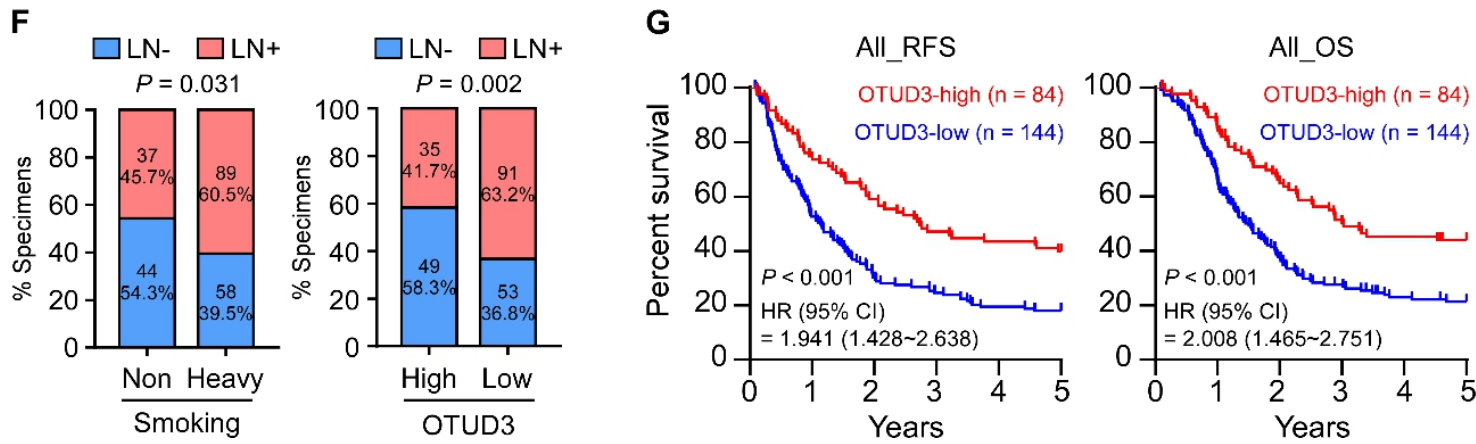

Figure 1. Nicotine-mediated OTUD3 downregulation correlates with lymph node metastasis and poor prognosis. (A) KYSE180 cells were treated with DMSO or cigarette smoke extract (CSE) $(100 \mu \mathrm{g} / \mathrm{ml})$ for $24 \mathrm{~h}$ and then subjected to RNA extraction and RNA-seq analysis. A volcanic plot showed the alterations of deubiquitinase (DUB) genes under CSE treatment. (B) qRT-PCR analysis of OTUD3 mRNA in the normal 
esophageal epithelial cell (NEEC) and six esophageal cancer cell lines with vehicle or CSE treatment for 24

h. (C) qRT-PCR analysis of OTUD3 mRNA in KYSE180 and KYSE520 cells treated with indicated tobacco carcinogens. (D) Representative images of OTUD3 IHC staining in normal $(n=5)$ and esophageal cancer specimens from non-smoking $(\mathrm{n}=81)$ and heavy-smoking $(\mathrm{n}=147)$ patients (left panel). The distribution of OTUD3 staining was compared (right panel). $\chi^{2}$ test was used. (E) The image (left panel) and quantification (right panel) of LYVE-1-positive lymphatic vessels in OTUD3-low or OTUD3-high specimens. (F) Correlation analyses between LN metastasis status and smoking history or OTUD3 expression in patient specimen. (G) Relapse-free survival (RFS) and overall survival (OS) analysis in esophageal cancer patients stratified by low and high OTUD3 expression $(n=228$, log-rank test) were shown. HR, hazard ratio. 
Figure 2

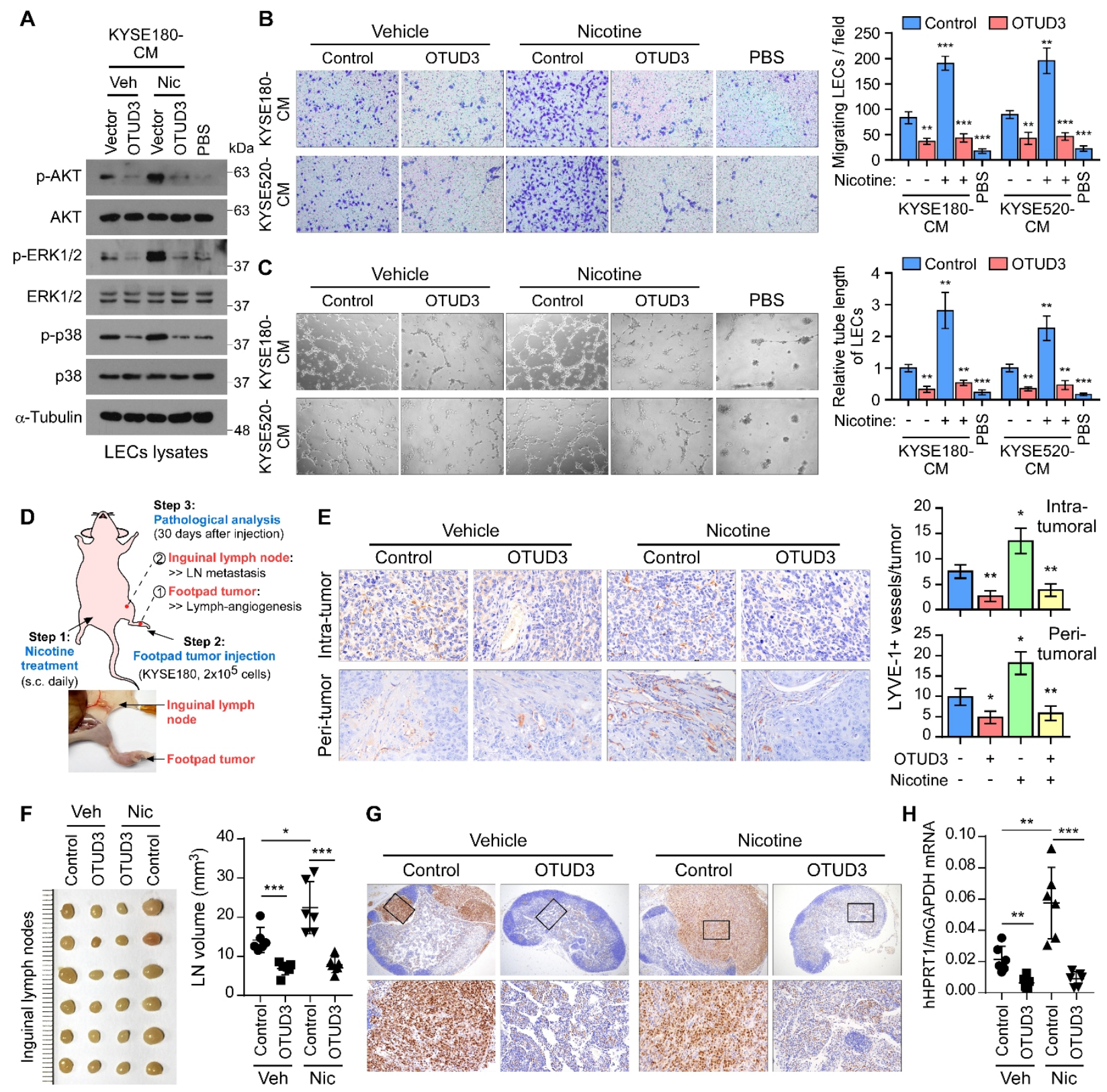

Figure 2. OTUD3 downregulation is required for nicotine-induced lymphatic metastasis. (A) Western blot analysis examined the phosphorylated levels of AKT, ERK1/2, and p38 in lymphatic endothelial cells (LECs) treated with indicated conditioned medium (CM). PBS was used as a negative control. (B) LECs incubated with indicated CM were subjected to transwell migration assays. (C) Tube formation assays of LECs under treatment of indicated CM. (D) Scheme procedure of nicotine administration and lymphatic metastasis examination in the mouse model. (E) Representative images and quantification of intra-tumoral and peri-tumoral lymphatic vessels in each group. (F) Image and volumes of the inguinal lymph nodes (LNs) from each group. (G) Metastatic KYSE180 cells in LNs were analyzed by the IHC staining of squamous cell carcinoma marker p63. (H) qRT-PCR analysis of human HPRT1 relative to mouse GAPDH in the LNs from each group. The ratio indicated the proportion of colonization. 


\section{Figure 3}

A

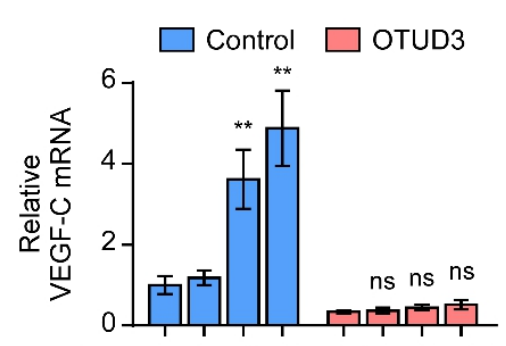

Nicotine: $0244872 \quad 0 \quad 244872$ h
B

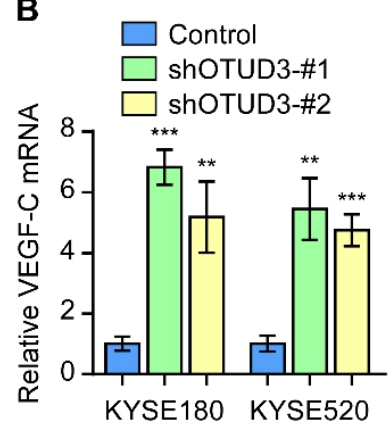

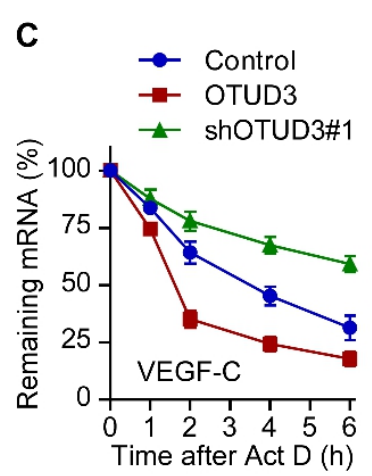

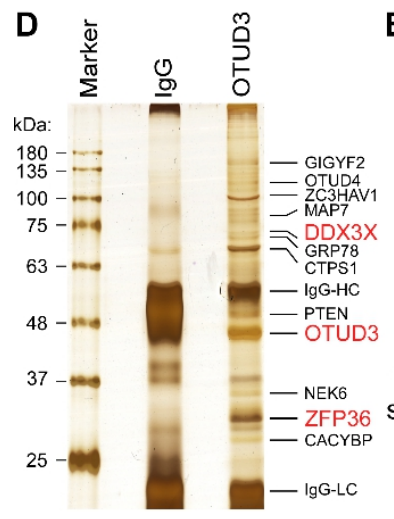

E

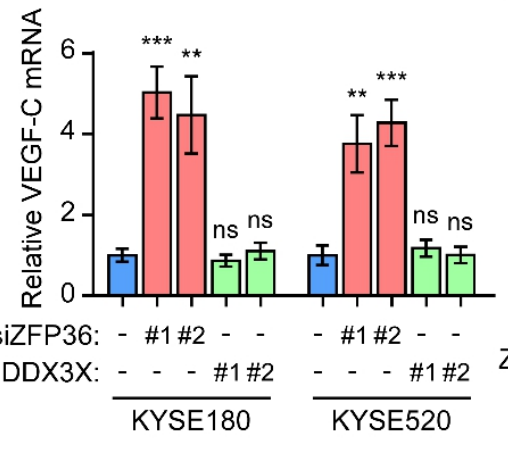

$\mathbf{F}$

年

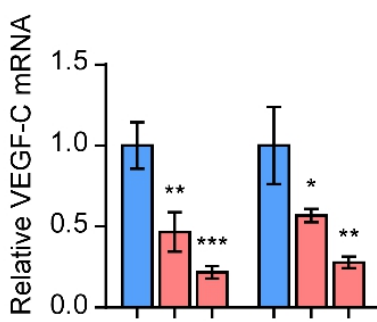

ZFP36 (ng): $020100 \quad 020100$

$\left.\overline{\text { KYSE180 }} \overline{\text { KYSE520 }} \grave{z}\right|_{\alpha \text {-Tubulin }}$

G

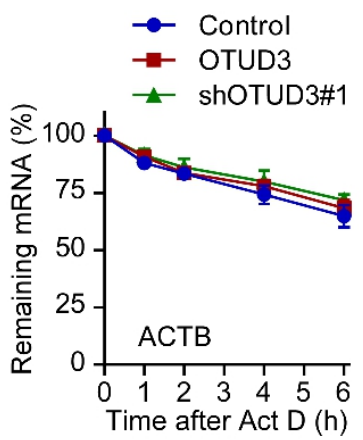

$\frac{\text { Veh }}{\overline{\text { on }} \frac{\text { Nic }}{\bar{c}}}$

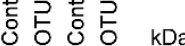

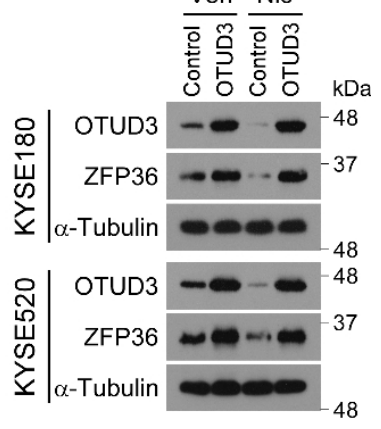

H

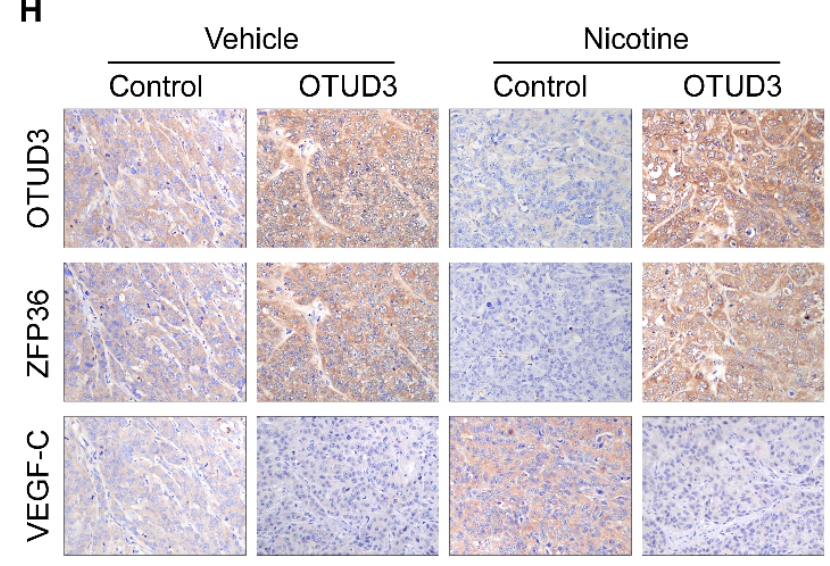

I

I
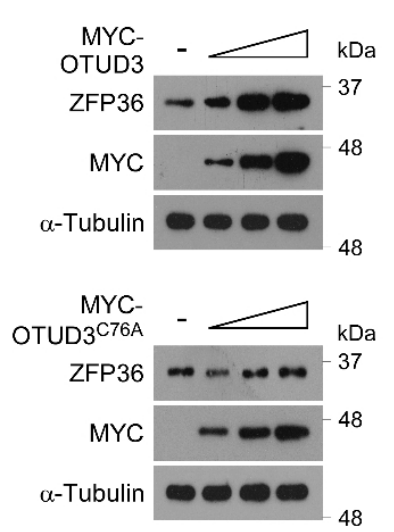

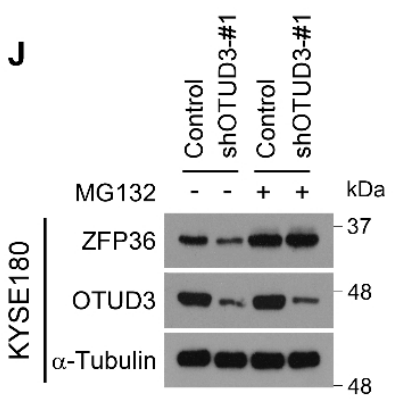

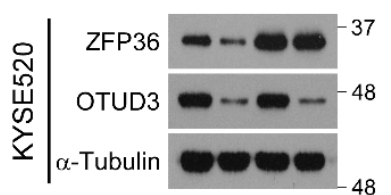

K
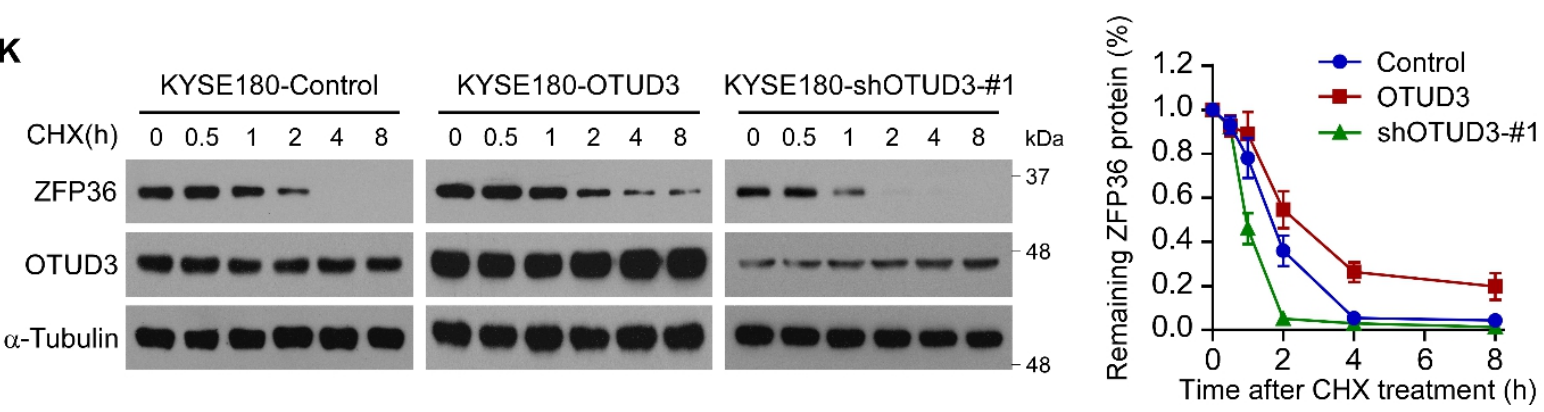

Figure 3. Nicotine promotes VEGF-C expression through downregulation of OTUD3 and ZFP36. (A) Control or OTUD3-overexpressing KYSE1 80 cells were treated with vehicle or nicotine for $24 \mathrm{~h}, 48 \mathrm{~h}$, and $72 \mathrm{~h}$, respectively. RNA was extracted and then subjected to qRT-PCR analysis of VEGF-C. (B) qRT-PCR analysis of VEGF-C in control and OTUD3 silencing esophageal cancer cells. (C) Control, 
OTUD3-expressing, or OTUD3-silencing KYSE180 cells were treated with Act D ( $5 \mu \mathrm{g} / \mathrm{ml})$. RNA was isolated at the indicated time points and then subjected to qRT-PCR analysis of VEGF-C and ACTB. The half-lives of mRNAs were traced by calculating the remaining mRNA levels relative to the untreated cells. (D) Immunoprecipitation of OTUD3 interacting proteins was subjected to SDS-PAGE, silver-stained, and identified by mass spectrometry (MS). (E) qRT-PCR analysis of VEGF-C in esophageal cancer cells transfected with NC, ZFP36, or DDX3X siRNAs. (F) qRT-PCR analysis of VEGF-C in esophageal cancer cells transfected with increasing ZFP36. (G) Western blot analysis of OTUD3 and ZFP36 in indicated cells. (H) IHC staining of OTUD3, ZFP36, and VEGF-C in the indicated footpad tumors. (I) Western blot analysis of ZFP36 and OTUD3 in KYSE180 cells with increasing transfection of MYC-OTUD3 or MYC-OTUD3 ${ }^{\mathrm{C} 76 \mathrm{~A}}$. (J) Western blot analysis of ZFP36 and OTUD3 in control and OTUD3-silencing esophageal cancer cells with or without MG132 treatment. (K) Indicated KYSE180 cells were treated with $100 \mu \mathrm{g} / \mathrm{ml}$ cycloheximide (CHX). Proteins were collected at the indicated time points and then immunoblotted with anti-ZFP36 and anti-OTUD3 antibodies. Quantification of ZFP36 protein levels was determined by normalizing to $\alpha$-tubulin protein. 
Figure 4
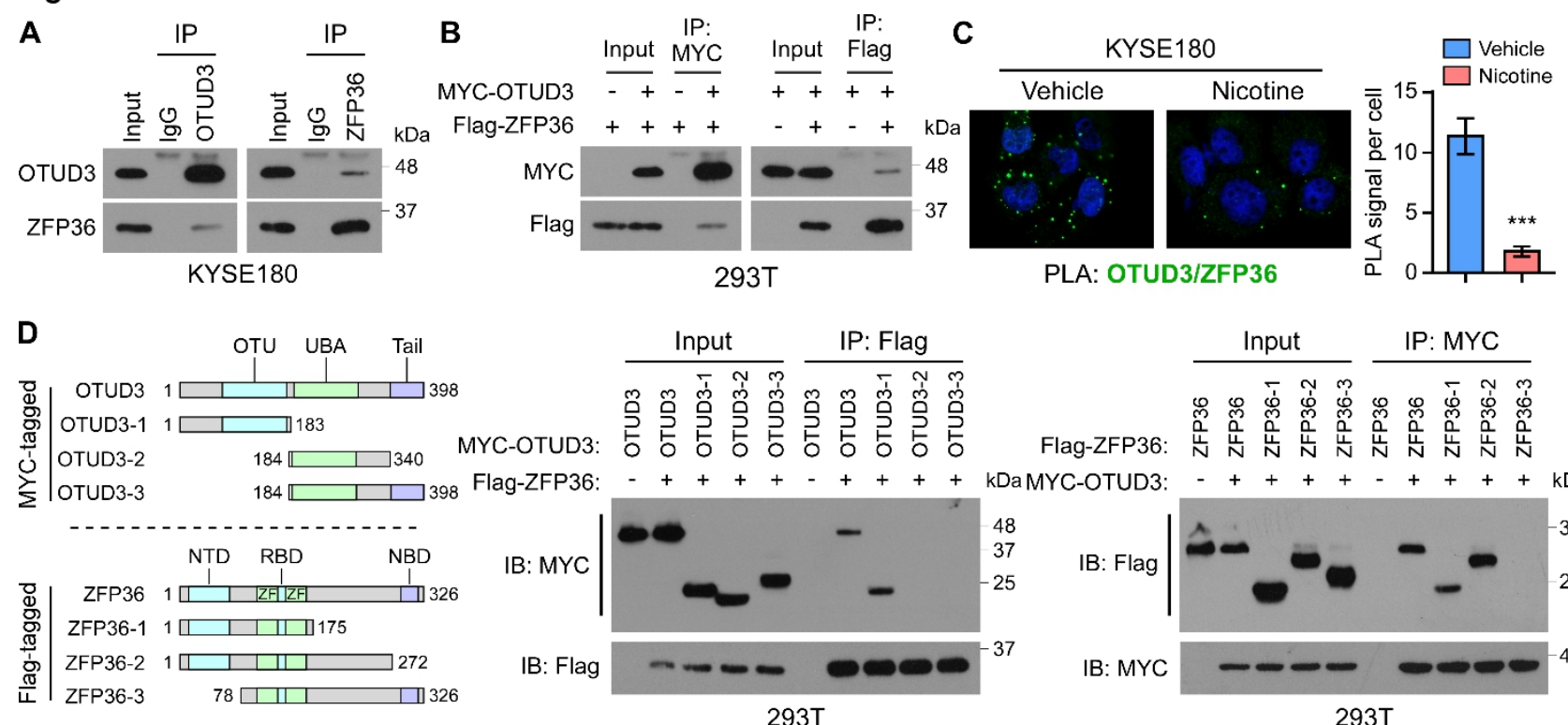

E

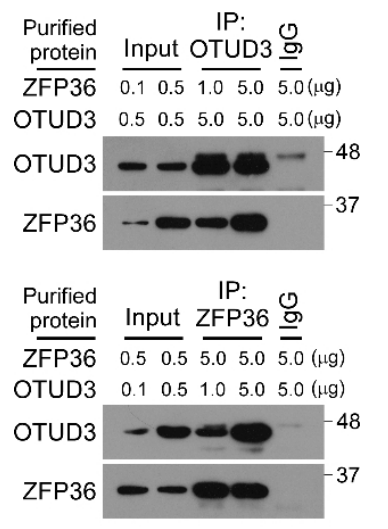

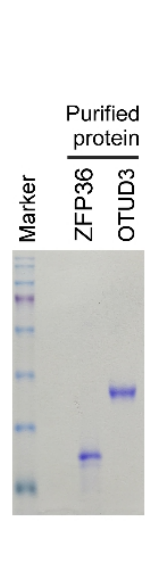

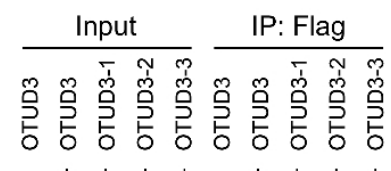

ag-ZFP36:

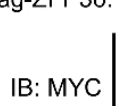

IB: MYC

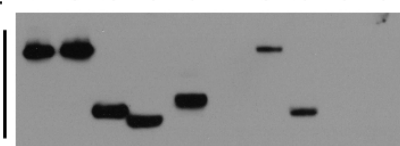

IB: Flag

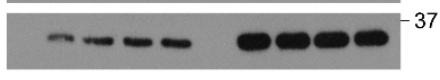

293T

$\mathbf{F}$

G
Input IP: MYC

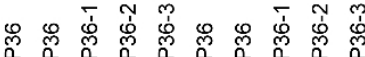

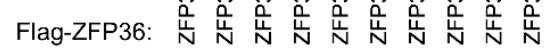
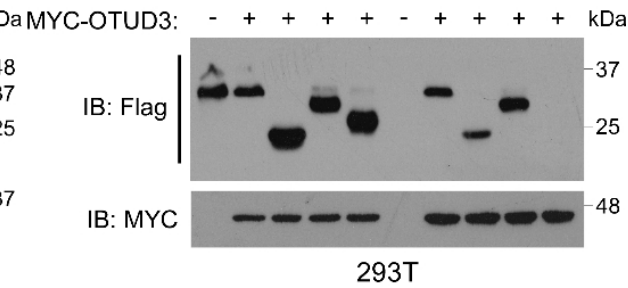

\section{$293 T$}

MG132 - + + + MG132 + + +

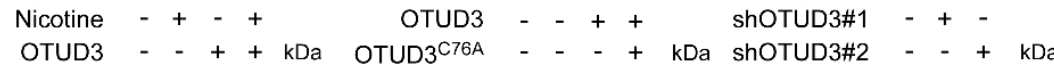

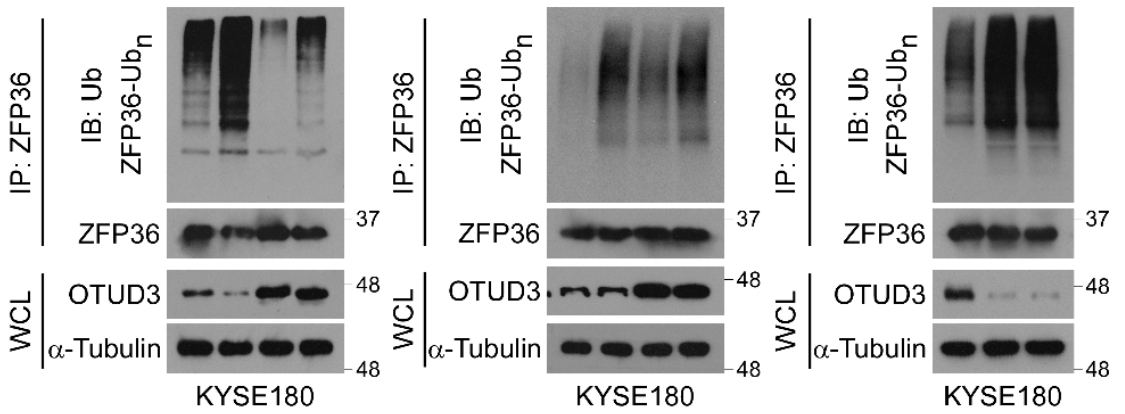

H

MG132 + + + +

$\mathrm{HA}-\mathrm{Ub}^{\mathrm{wt}}++-\cdot$

$\mathrm{HA}-\mathrm{Ub}^{\mathrm{K} 48}-\boldsymbol{H}^{-}+$

MYC-OTUD3 - + + $\mathrm{kDa}$

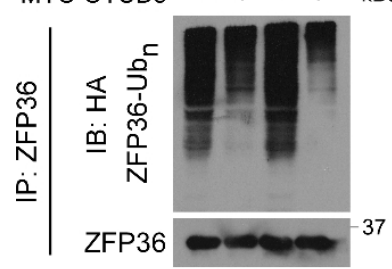

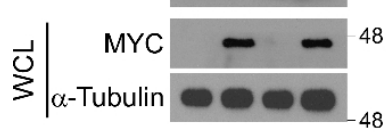

293T

I

MG132 + + +

FBXW7 - + +

OTUD3

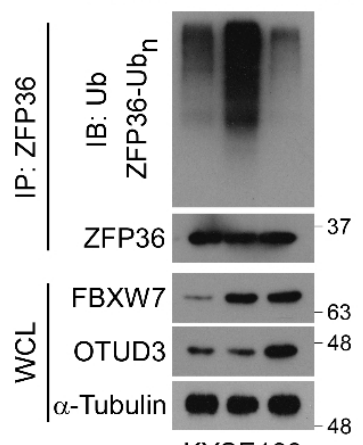

KYSE180

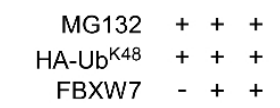

FBXW7 -+
MYC-OTUD3 -+
-

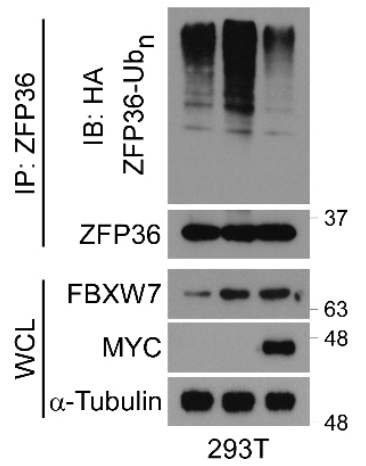

J

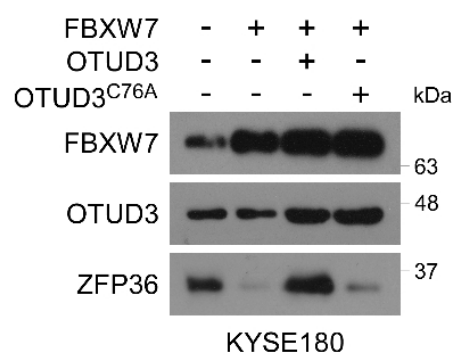

Figure 4. OTUD3 inhibits the K48-linked ubiquitination and degradation of ZFP36. (A) Reciprocal immunoprecipitation (IP) assays of OTUD3 and ZFP36 in KYSE180 cells. (B) The 293T cells were transfected with MYC-OTUD3 and Flag-ZFP36 constructs, followed by IP assays using MYC- or Flag-conjugated agarose. (C) Proximity ligation assay indicated the interaction between OTUD3 and ZFP36 
in KYSE180 cells treated with vehicle or nicotine. (D) Schematic illustration of OTUD3 and ZFP36 truncated constructs was shown. IP assays were performed to examine the interactions. (E) In vitro protein binding assays with increasing dose of purified OTUD3 and ZFP36 proteins. The purities of OTUD3 and ZFP36 were examined by SDS-PAGE and Coomassie Blue Staining. (F) Control or OTUD3-overexpressing KYSE180 cells were treated with or without nicotine treatment. Cells were treated with MG132 $(10 \mu \mathrm{M})$ for $6 \mathrm{~h}$ before harvest. Lysates were then immunoprecipitated with anti-ZFP36 antibody, followed by immunoblotting with anti-Ub and anti-ZFP36 antibodies to examine the ubiquitination levels. WCL: whole cell lysate. (G) OTUD3-overexpressing or -silencing KYSE180 cells were treated with MG132 $(10 \mu \mathrm{M})$, and then subjected to ZFP36 ubiquitination analysis. (H) The 293T cells were transfected with HA-Ub ${ }^{\mathrm{wt}}$, HA-Ub ${ }^{\mathrm{K} 48}$, and MYC-OTUD3. Lysates were then subjected to ZFP36 ubiquitination analysis. (I) Analysis of ZFP36 endogenous (left) or K48-linked (right) ubiquitination in KYSE180 cells transfected with FBXW7, or FBXW7 plus OTUD3. (J) Western blot analysis of FBXW7, OTUD3, and ZFP36 in indicated KYSE180 cells. 


\section{Figure 5}

A

human VEGF-C transcript

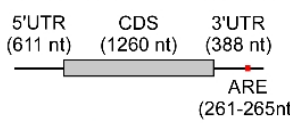

5'-tgactatataatttatttccactaa-3' Mus.M 5'-tgactatataatttatttccattaa-3' Pan $T$

5'-tgactatataatttattccactaa- 3 ' Bos. $T$

5'-tgactatataatttatttccactaa-3' Homo.S

5'-tgactatataaaaaatttccactaa-3' ARE-mut
B

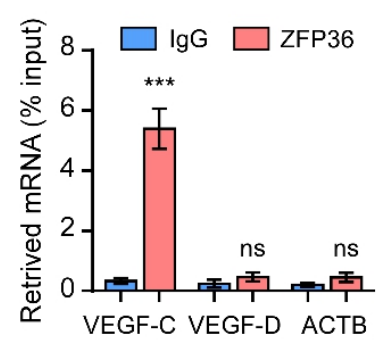

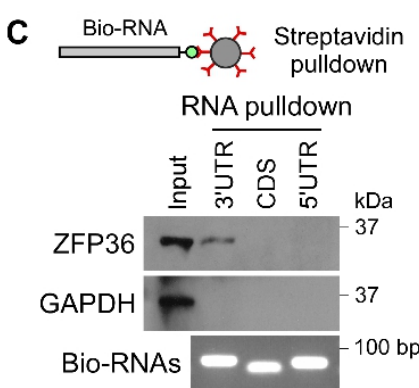

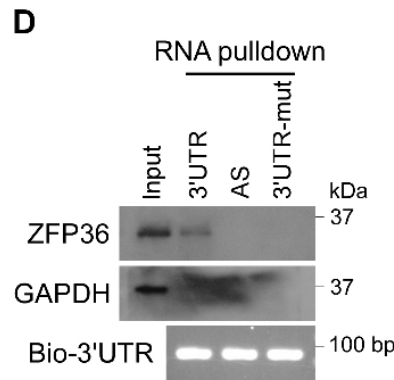

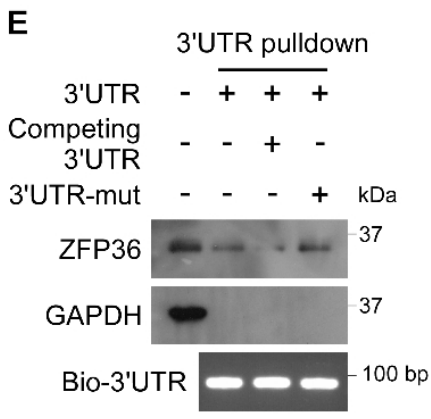
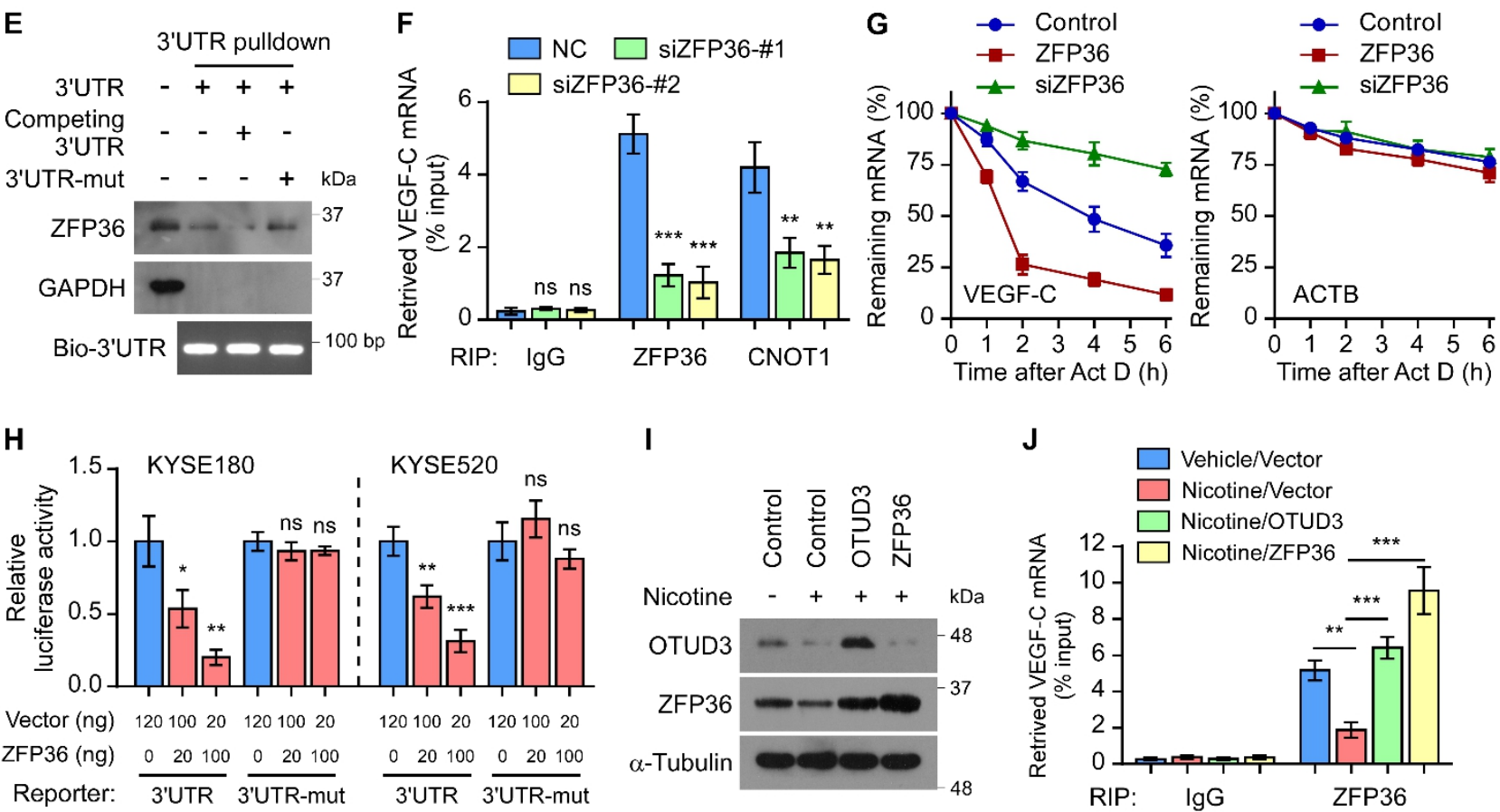

I
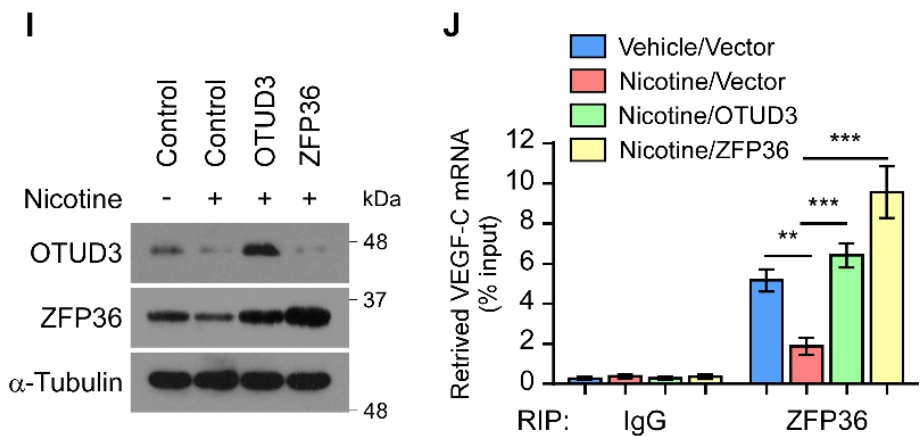

K

$\mathbf{L}$
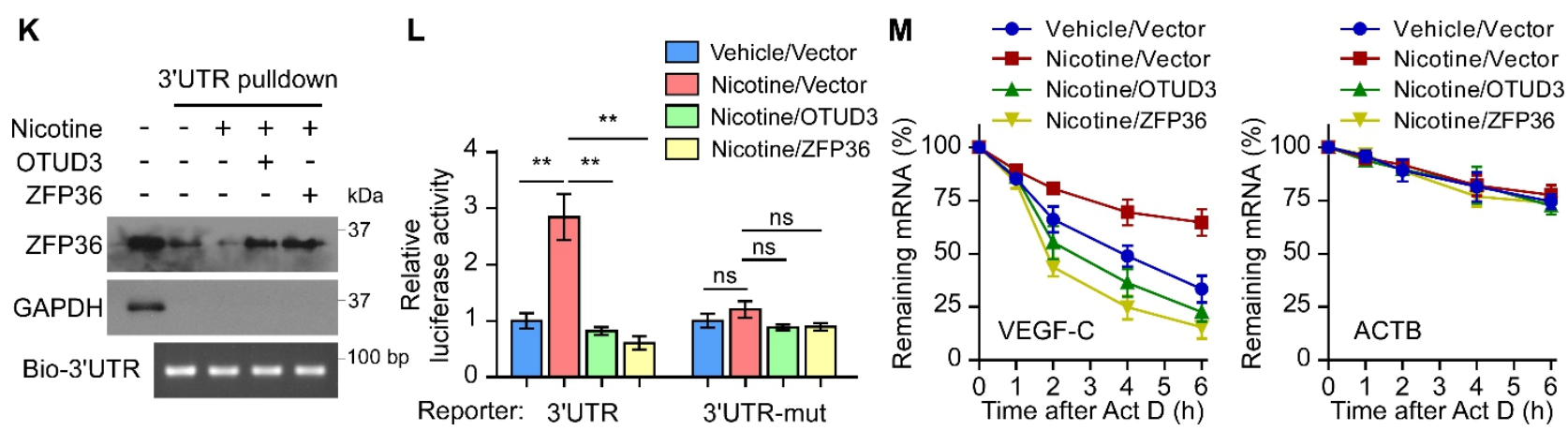

Figure 5. Nicotine inhibits VEGF-C mRNA decay. (A) The VEGF-C 3'UTR contained a conserved AU-rich element (ARE) among species. (B) RNA immunoprecipitation (RIP) assay was performed in KYSE180 cells using an anti-ZFP36 antibody. Retrieved RNA was then subjected to qRT-PCR analysis of VEGF-C, VEGF-D, and ACTB. IgG was used as a negative control. (C) In vitro-transcribed and biotin-labeled VEGF-C 5'UTR, CDS, and 3'UTR RNAs were incubated with KYSE180 lysates, followed by streptavidin pulldown and detection of ZFP36. GAPDH was used as a negative control. (D) Western blot analysis of ZFP36 following the streptavidin pulldown of biotin-labeled VEGF-C 3'UTR, 3'UTR-ARE-mut, 
or 3'UTR-AS in KYSE180 lysates. (E) Streptavidin pulldown of biotin-labeled VEGF-C 3'UTR with or without competing 3'UTR (unlabeled) or bio-3'UTR-mut. The interaction of ZFP36 protein was examined by western blot. (F) RIP assays of ZFP36 and CNOT1 followed by the qRT-PCR analysis of VEGF-C in control or ZFP36-silencing KYSE180 cells. (G) The half-life of VEGF-C mRNA was traced in indicated KYSE180 cells. ACTB was used as a negative control. (H) Luciferase reporters of VEGF-C 3'UTR and 3'UTR-mut in indicated KYSE180 cells. (I) Western blot analysis of OTUD3 and ZFP36 in KYSE180 cells with or without nicotine treatment. (J) RIP assays of ZFP36 followed by the qRT-PCR analysis of VEGF-C in indicated KYSE180 cells with or without nicotine treatment. (K) Streptavidin pulldown of biotin-labeled VEGF-C 3'UTR in indicated KYSE180 lysates, followed by ZFP36 detection. (L) Luciferase reporters of VEGF-C 3'UTR or 3'UTR-mut in indicated KYSE180 cells. (M) VEGF-C mRNA's half-life was traced in indicated KYSE180 cells with or without nicotine treatment. 
Figure 6
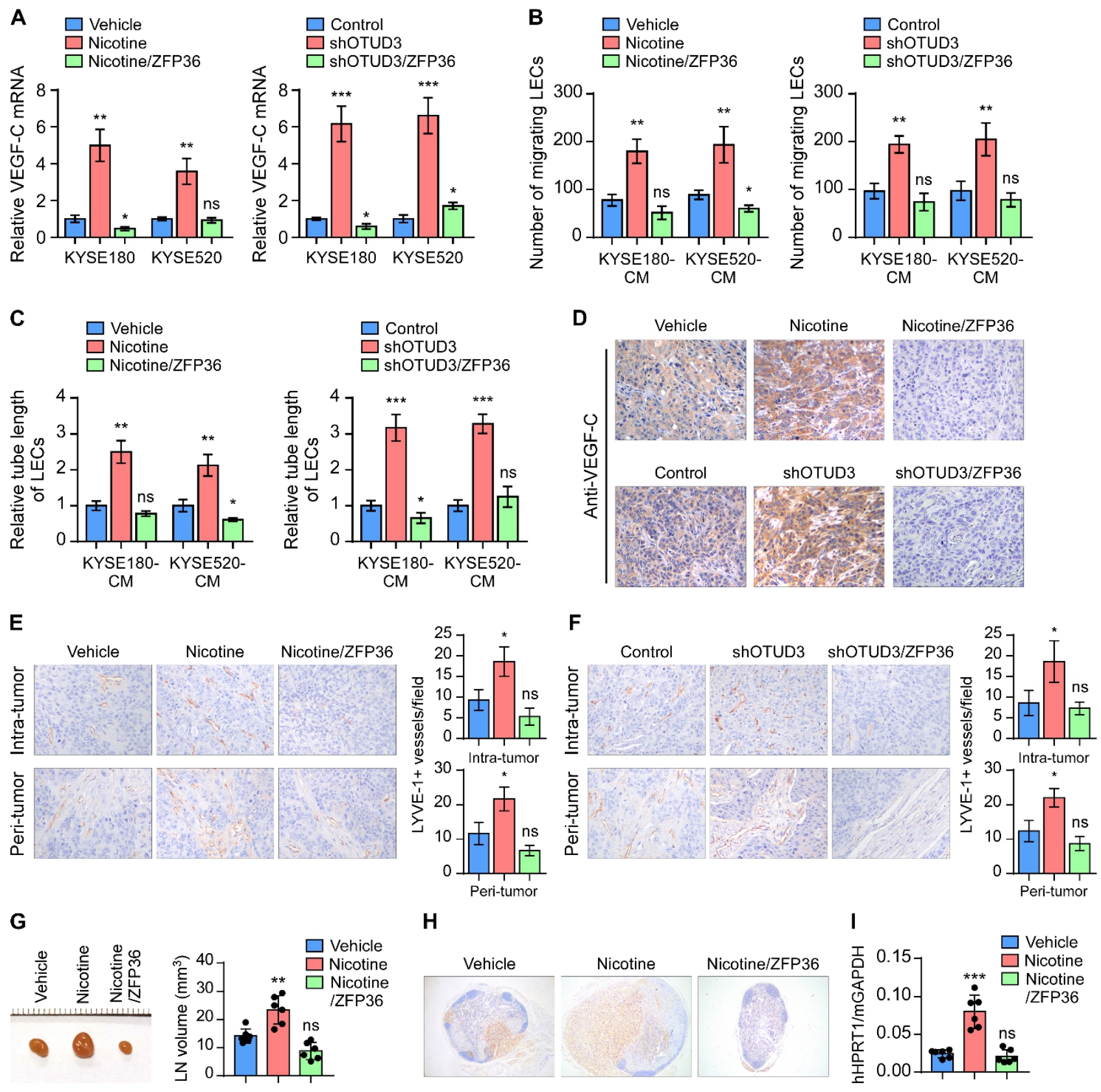

H
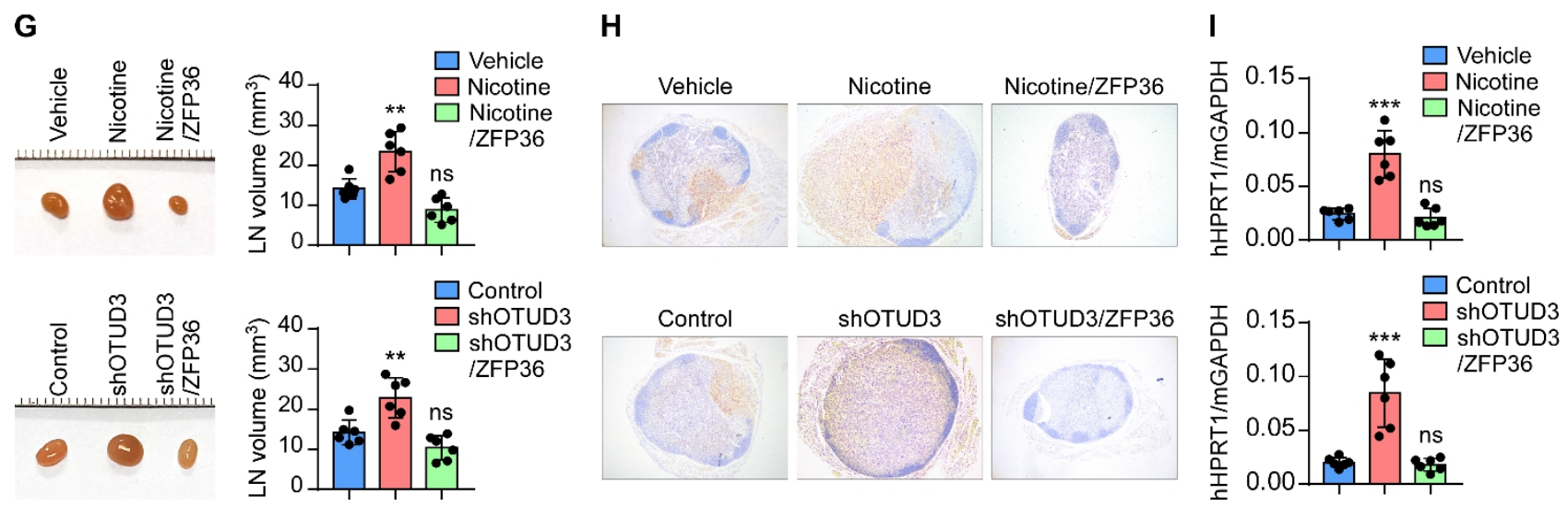

Figure 6. Induction of VEGF-C mRNA decay abrogates lymphatic metastasis. (A) qRT-PCR analysis of VEGF-C mRNA expression in indicated esophageal cancer cells. (B) Quantification of migrating LECs under treatment of indicated CM. (C) Relative tube length of LECs under treatment of indicated CM. (D) IHC staining of VEGF-C in footpad tumors. (E-F) Representative images of LYVE-1 staining and quantification of intra-tumoral (E) and peri-tumoral (F) lymphatic vessels in indicated footpad tumors. (G) 
Representative image of inguinal LNs. The volumes of LNs from each group were quantified. (H) IHC staining of squamous cell carcinoma marker p63 indicated the metastatic KYSE180 cells in LNs. (I) qRT-PCR analysis of human HPRT1 relative to mouse GAPDH in the LNs from each group. 
Figure 7
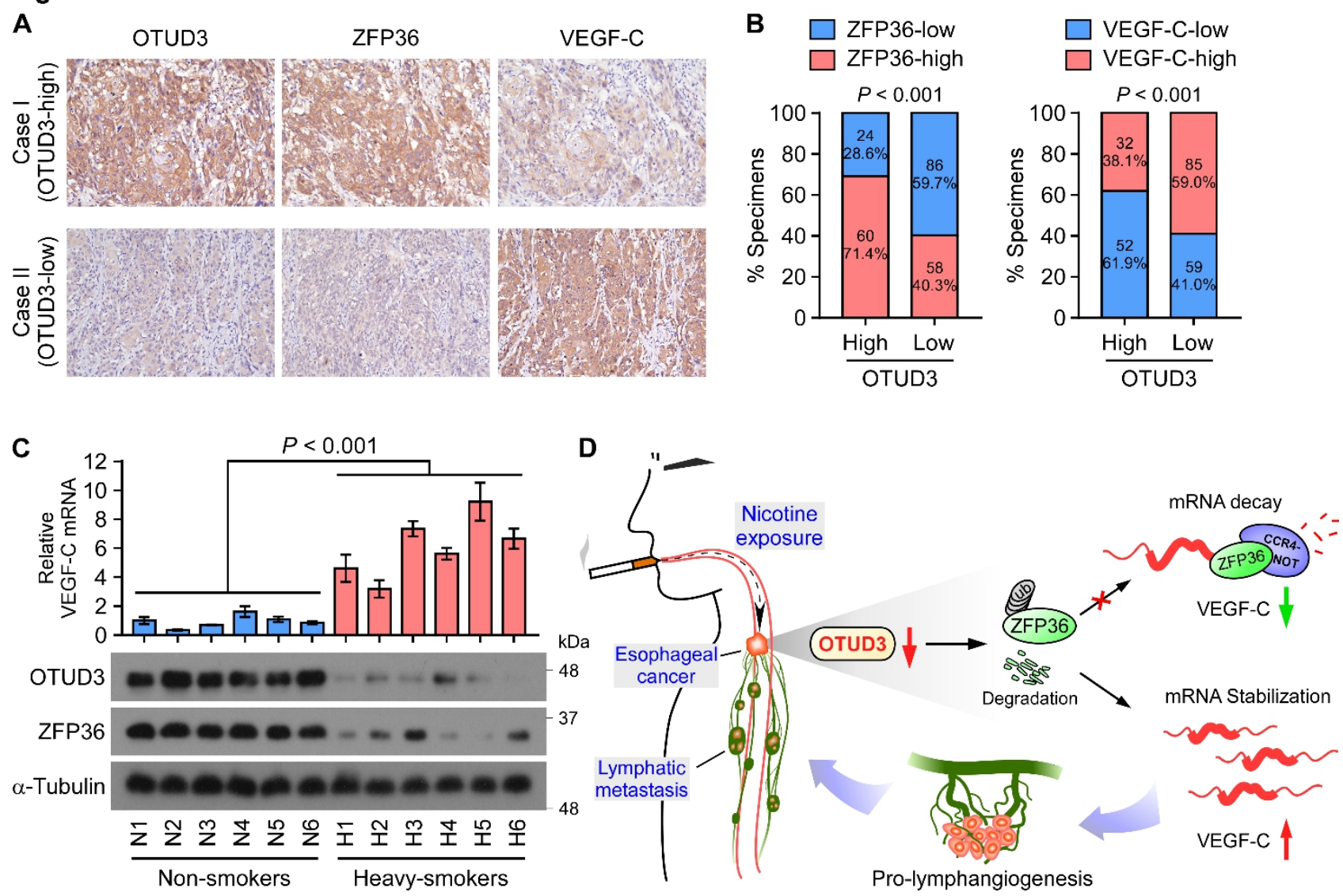

Figure 7. Clinical relevance and study model. (A) Representative IHC staining images of OTUD3, ZFP36, and VEGF-C in esophageal cancer patient specimens $(n=228)$. (B) Correlation analysis showed that OTUD3 was significantly associated with ZFP36 and VEGF-C expression. $\chi^{2}$ test was used. (C) qRT-PCR of VEGF-C and western blot analysis of OTUD3 and ZFP36 in primary esophageal tumors derived from 6 non-smoking and 6 heavy-smoking patients. (D) Study model: Nicotine-mediated OTUD3 downregulation facilitates ZFP36 protein degradation and inhibits VEGF-C mRNA decay, leading to robust VEGF-C production, tumor-induced lymphangiogenesis, and lymphatic metastasis in esophageal cancer. 
Figures

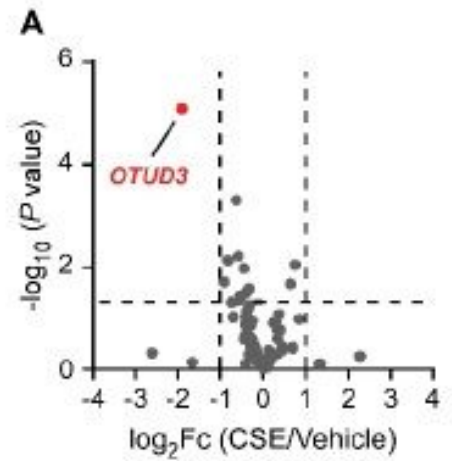

B

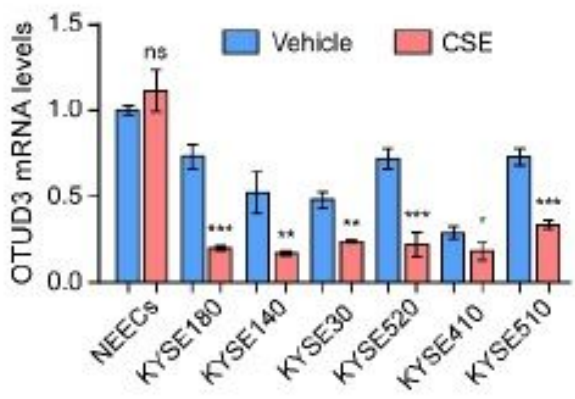

C

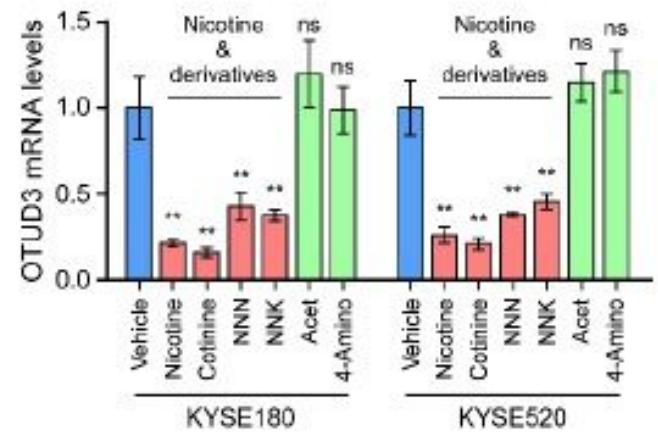

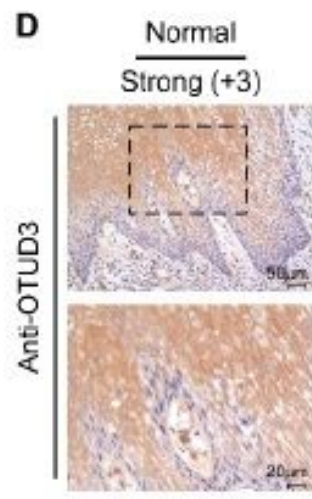

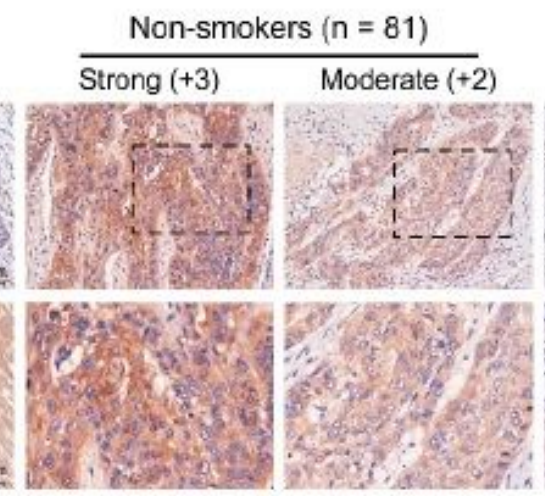

\section{$\frac{\text { Heavy-sm }}{\text { Weak }(+1)}$}

$\frac{\text { okers }(n=147)}{\text { Negative }(0)}$

E
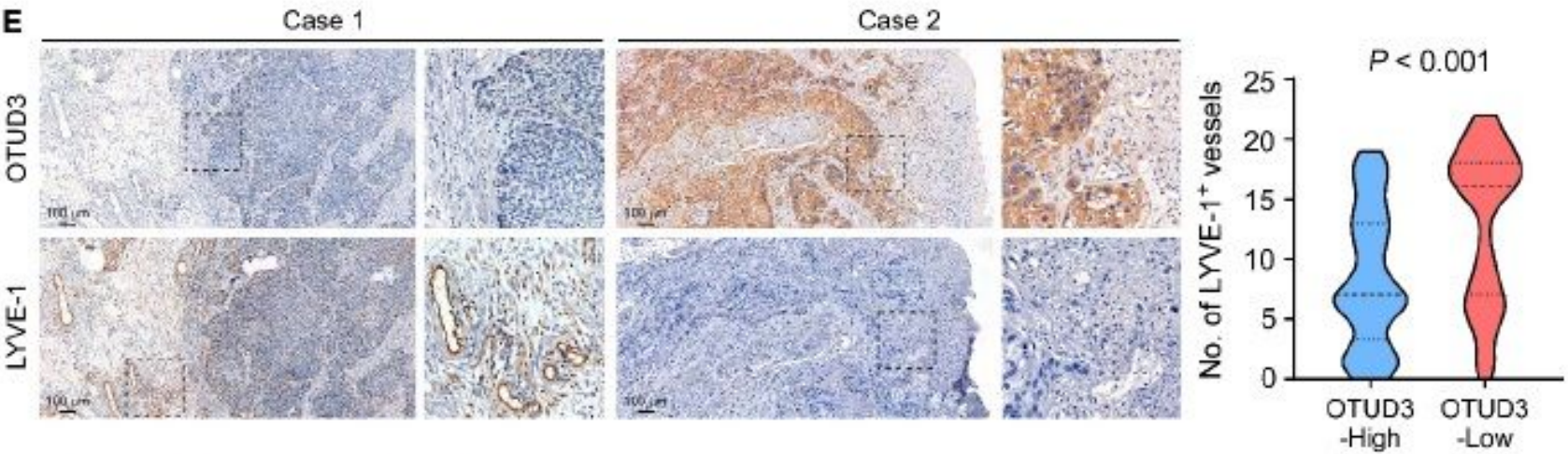

$\mathbf{F}$

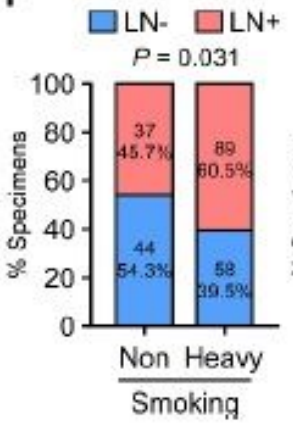

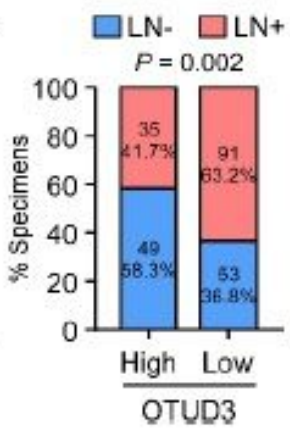

G

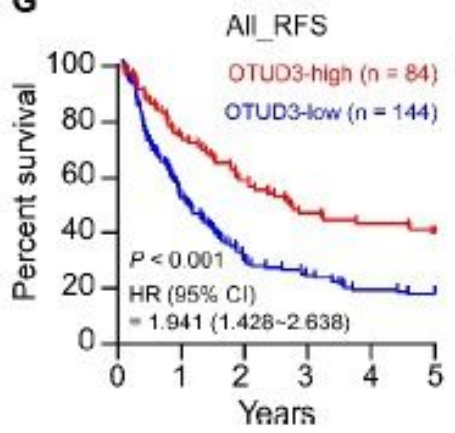

$\square$ Nagative $\square$ Moderate

$\square$ Weak $\square$ Strong

$7.41 \%$

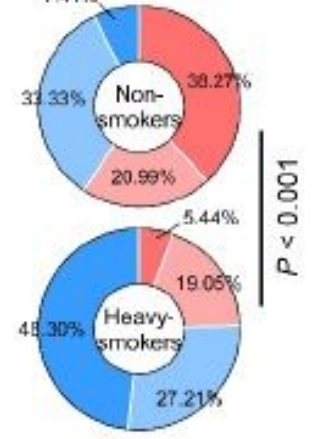

-High

All_OS

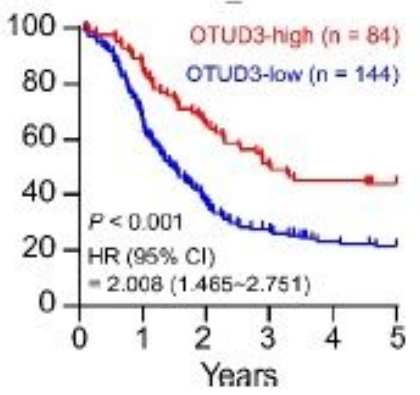

\section{Figure 1}

Nicotine-mediated OTUD3 downregulation correlates with lymph node metastasis and poor prognosis. (A) KYSE180 cells were treated with DMSO or cigarette smoke extract (CSE) $(100 \mu \mathrm{g} / \mathrm{ml})$ for $24 \mathrm{~h}$ and then subjected to RNA extraction and RNA-seq analysis. A volcanic plot showed the alterations of 
deubiquitinase (DUB) genes under CSE treatment. (B) qRT-PCR analysis of OTUD3 mRNA in the normal h. (C) qRT-PCR analysis of OTUD3 mRNA in KYSE180 and KYSE520 cells treated with indicated tobacco carcinogens. (D) Representative images of OTUD3 IHC staining in normal $(n=5)$ and esophageal cancer specimens from non-smoking $(n=81)$ and heavy-smoking $(n=147)$ patients (left panel). The distribution of OTUD3 staining was compared (right panel). $\chi 2$ test was used. (E) The image (left panel) and quantification (right panel) of LYVE-1-positive lymphatic vessels in OTUD3-low or OTUD3-high specimens. (F) Correlation analyses between LN metastasis status and smoking history or OTUD3 expression in patient specimen. (G) Relapse-free survival (RFS) and overall survival (OS) analysis in esophageal cancer patients stratified by low and high OTUD3 expression $(n=228$, log-rank test) were shown. HR, hazard ratio.

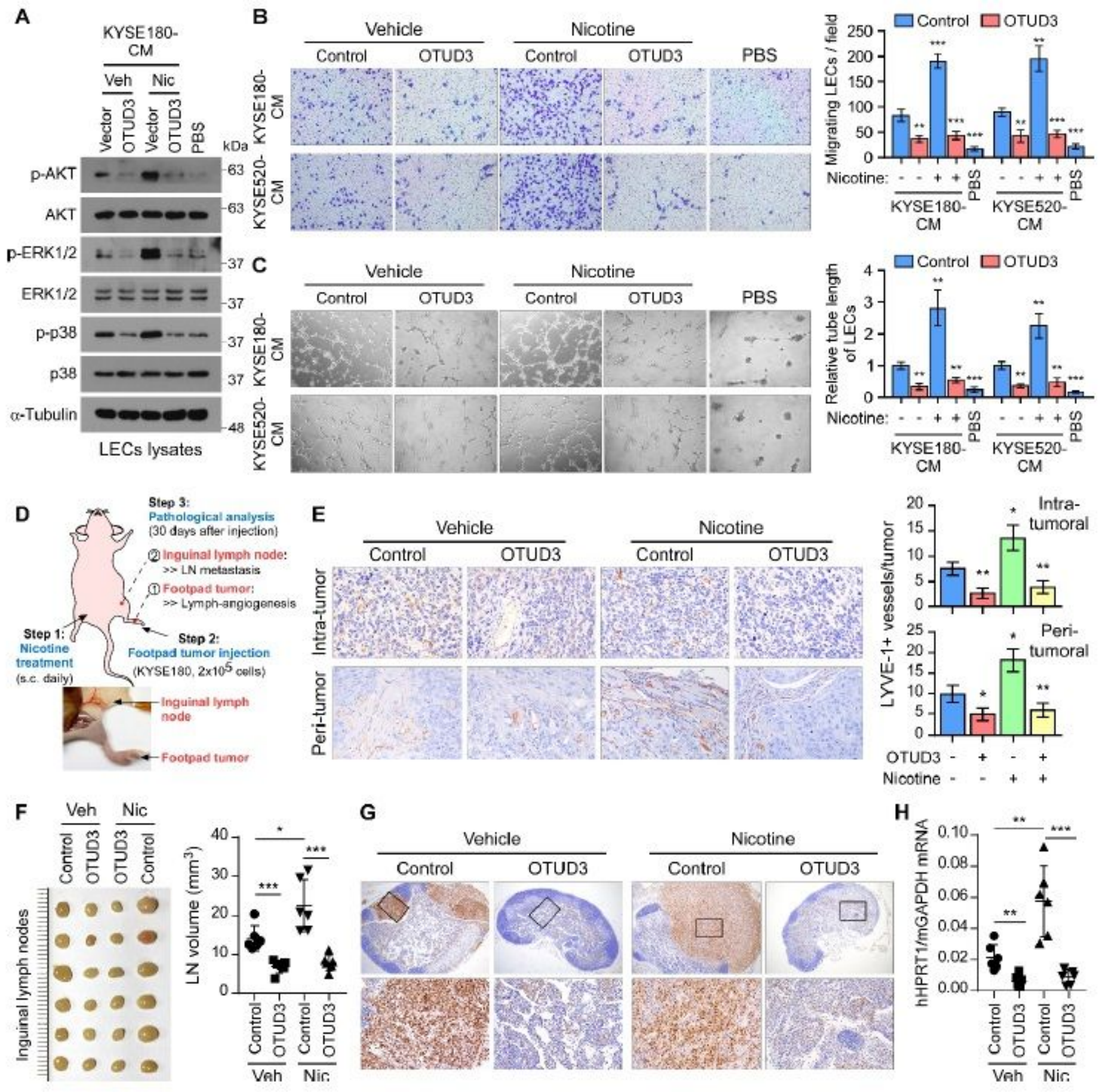




\section{Figure 2}

OTUD3 downregulation is required for nicotine-induced lymphatic metastasis. (A) Western blot analysis examined the phosphorylated levels of AKT, ERK1/2, and p38 in lymphatic endothelial cells (LECs) treated with indicated conditioned medium (CM). PBS was used as a negative control. (B) LECs incubated with indicated CM were subjected to transwell migration assays. (C) Tube formation assays of LECs under treatment of indicated CM. (D) Scheme procedure of nicotine administration and lymphatic metastasis examination in the mouse model. (E) Representative images and quantification of intratumoral and peri-tumoral lymphatic vessels in each group. (F) Image and volumes of the inguinal lymph nodes (LNs) from each group. (G) Metastatic KYSE180 cells in LNs were analyzed by the IHC staining of squamous cell carcinoma marker p63. (H) qRT-PCR analysis of human HPRT1 relative to mouse GAPDH in the LNs from each group. The ratio indicated the proportion of colonization. 
A

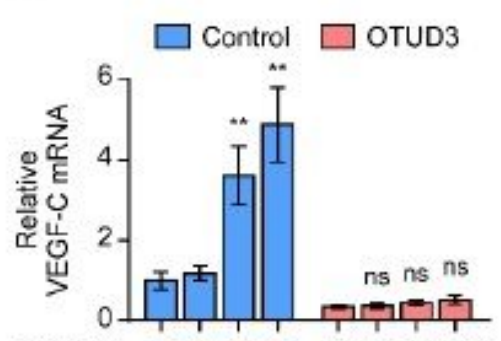

Nicotine: $0244872 \quad 0244872 \mathrm{~h}$
B
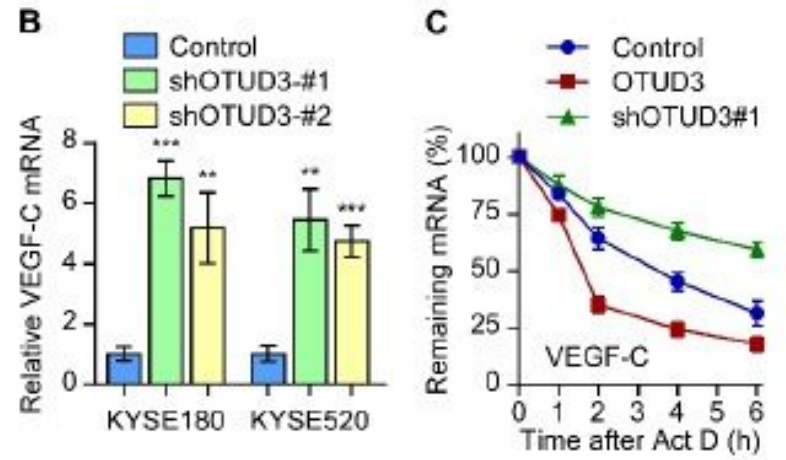

$\mathbf{F}$

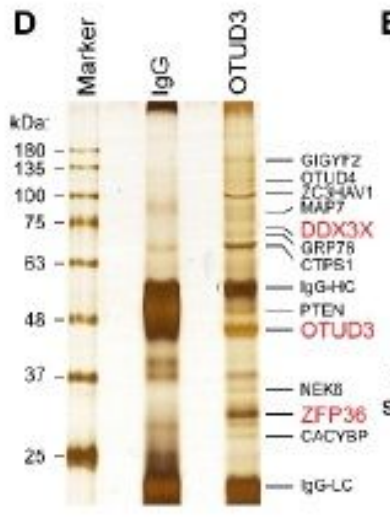

H

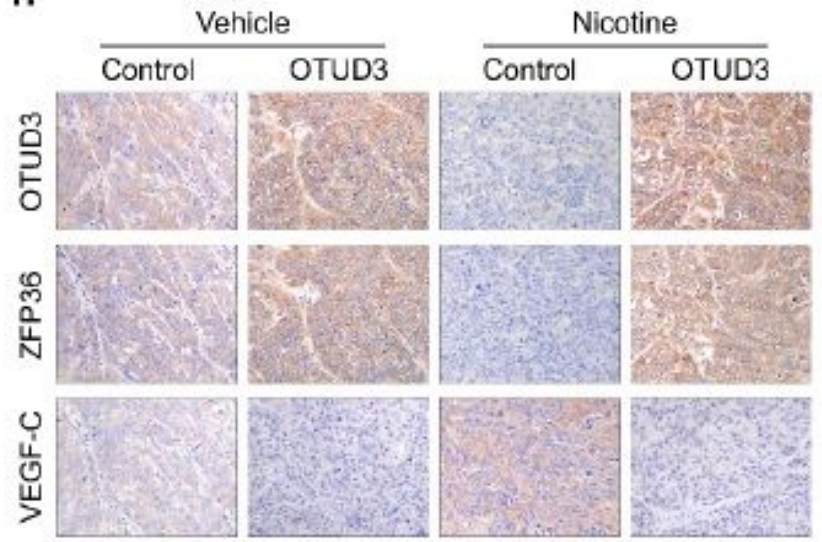

E

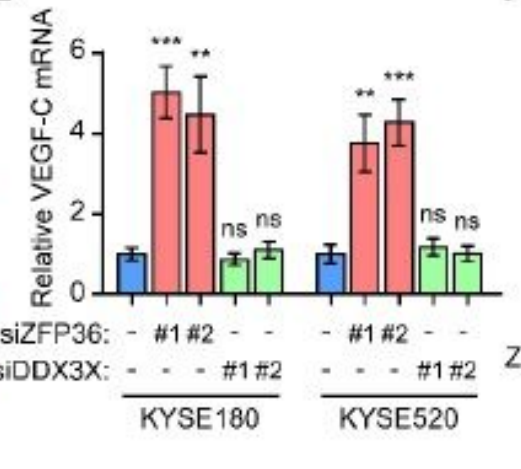

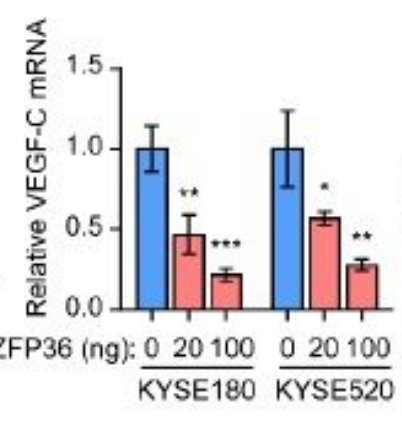

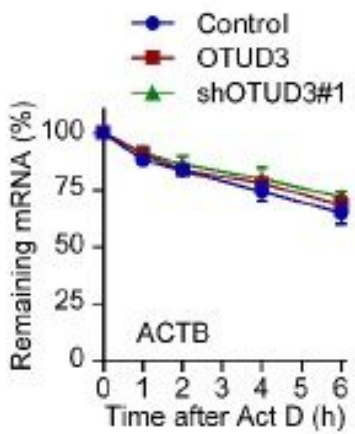

G

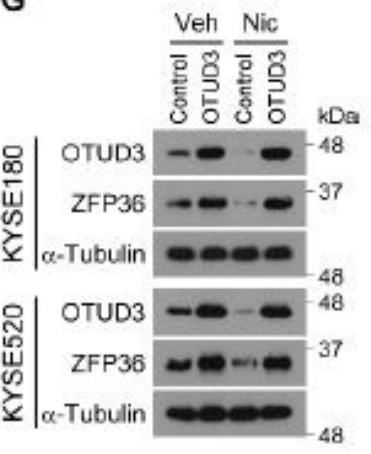

K
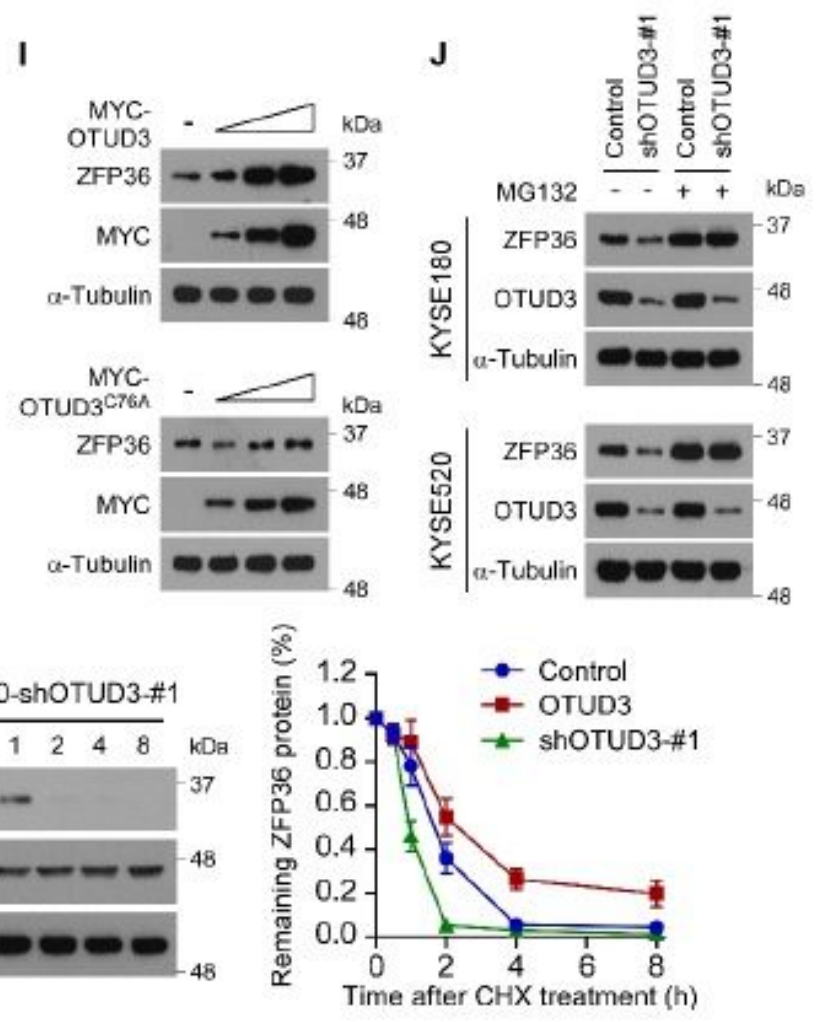

\section{Figure 3}

Nicotine promotes VEGF-C expression through downregulation of OTUD3 and ZFP36. (A) Control or OTUD3-overexpressing KYSE180 cells were treated with vehicle or nicotine for $24 \mathrm{~h}, 48 \mathrm{~h}$, and $72 \mathrm{~h}$, respectively. RNA was extracted and then subjected to qRT-PCR analysis of VEGF-C. (B) qRT-PCR analysis of VEGF-C in control and OTUD3 silencing esophageal cancer cells. (C) Control, OTUD3-expressing, or OTUD3-silencing KYSE180 cells were treated with Act D $(5 \mu \mathrm{g} / \mathrm{ml})$. RNA was isolated at the indicated time 
points and then subjected to qRT-PCR analysis of VEGF-C and ACTB. The half-lives of mRNAs were traced by calculating the remaining mRNA levels relative to the untreated cells.(D) Immunoprecipitation of OTUD3 interacting proteins was subjected to SDS-PAGE, silver-stained, and identified by mass spectrometry (MS). (E) qRT-PCR analysis of VEGF-C in esophageal cancer cells transfected with NC, ZFP36, or DDX3X siRNAs. (F) qRT-PCR analysis of VEGF-C in esophageal cancer cells transfected with increasing ZFP36. (G) Western blot analysis of OTUD3 and ZFP36 in indicated cells.(H) IHC staining of OTUD3, ZFP36, and VEGF-C in the indicated footpad tumors. (I) Western blot analysis of ZFP36 and OTUD3 in KYSE180 cells with increasing transfection of MYC-OTUD3 or MYC-OTUD3C76A. (J) Western blot analysis of ZFP36 and OTUD3 in control and OTUD3-silencing esophageal cancer cells with or without MG132 treatment. (K) Indicated KYSE180 cells were treated with $100 \mu \mathrm{g} / \mathrm{ml}$ cycloheximide (CHX). Proteins were collected at the indicated time points and then immunoblotted with anti-ZFP36 and antiOTUD3 antibodies. Quantification of ZFP36 protein levels was determined by normalizing to a-tubulin protein. 


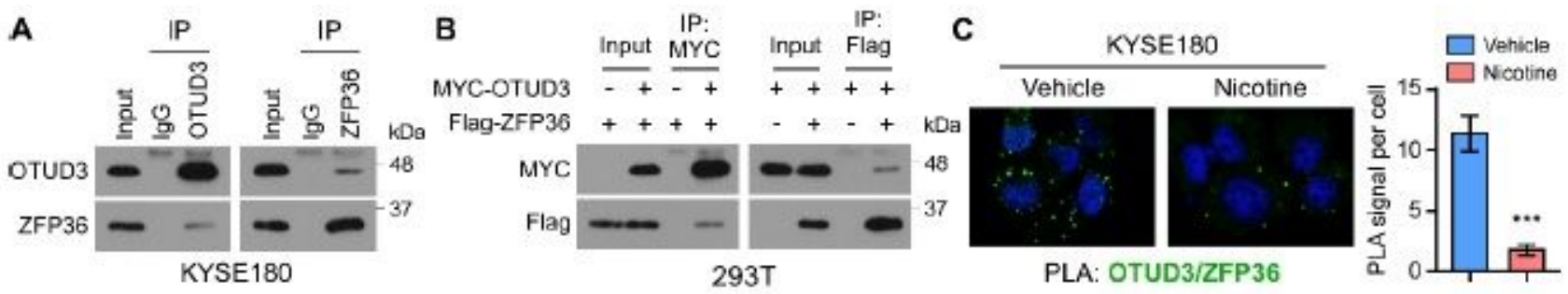

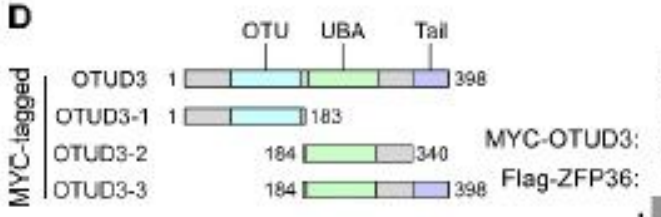

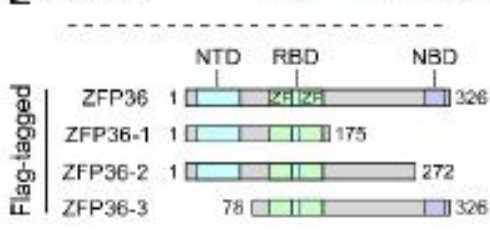

E

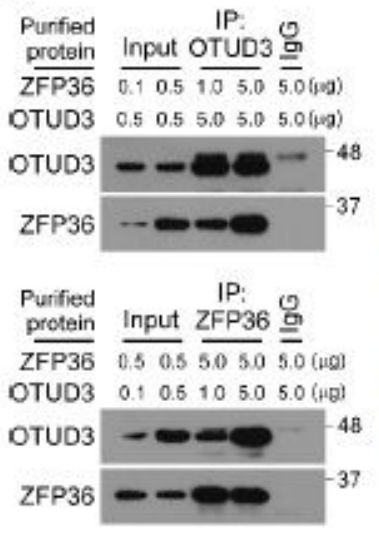

H

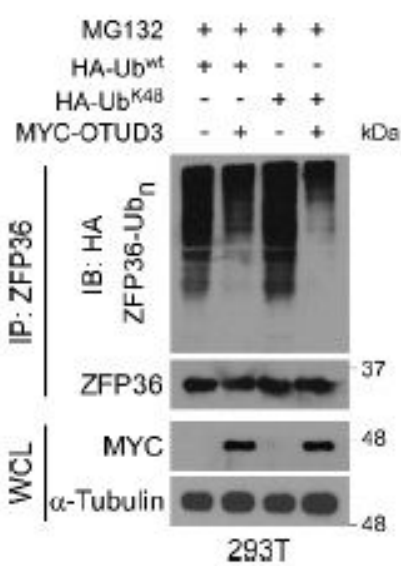

I

MG132 + + +

FBXW7 - + +
IB: MYC

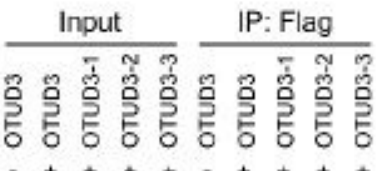

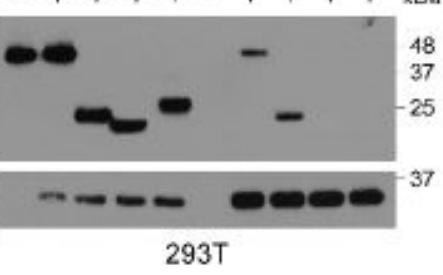

G

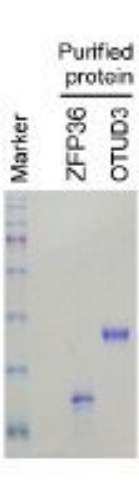

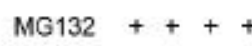

Nicotine - + + OTUD3 - $++k \mathrm{kB}$

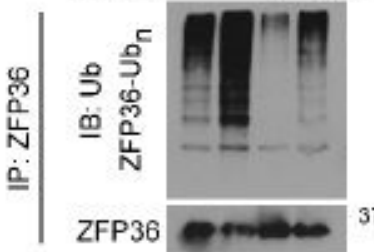

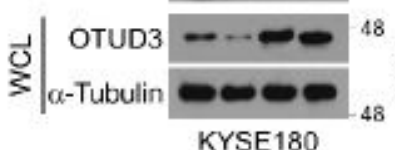

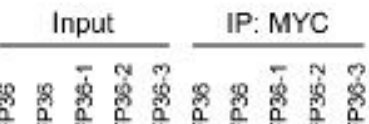

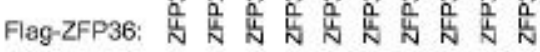

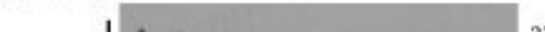

IB: MYC $-0--48$ 293T

IB: Fla

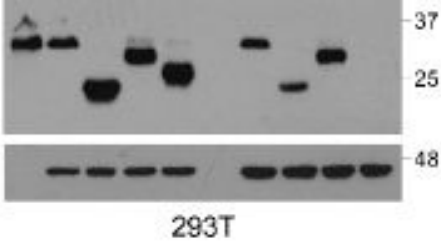

MG132 - + + + MG132 + + +

OTUD3 - ++ ShOTUD3H1 - + OTUD3 ${ }^{\mathrm{C} 784}$ - . + $+\mathrm{kDa}$ shOTUD3H2 - +

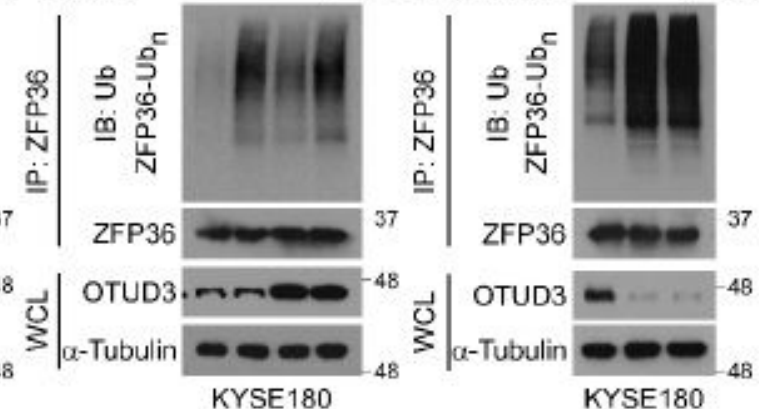

OTUD3 - + $+\mathrm{kDa}$

MG132 + + +

$\mathrm{HA}-\mathrm{Ub}^{\mathrm{K} 48}+++$

FBXW7 - + +

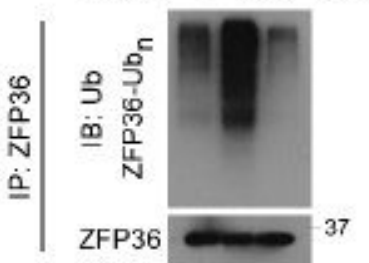

MYC-OTUD3 - + + kDa

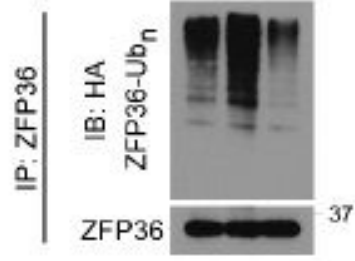

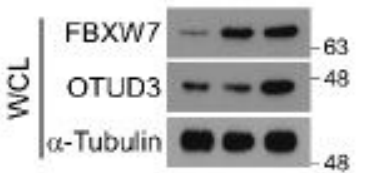

KYSE180

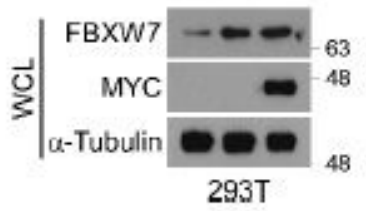

KYSE 180

\section{Figure 4}

OTUD3 inhibits the K48-linked ubiquitination and degradation of ZFP36. (A) Reciprocal immunoprecipitation (IP) assays of OTUD3 and ZFP36 in KYSE180 cells. (B) The 293T cells were transfected with MYC-OTUD3 and Flag-ZFP36 constructs, followed by IP assays using MYC- or Flagconjugated agarose. (C) Proximity ligation assay indicated the interaction between OTUD3 and ZFP36 in KYSE180 cells treated with vehicle or nicotine. (D) Schematic illustration of OTUD3 and ZFP36 truncated 
constructs was shown. IP assays were performed to examine the interactions. (E) In vitro protein binding assays with increasing dose of purified OTUD3 and ZFP36 proteins. The purities of OTUD3 and ZFP36 were examined by SDS-PAGE and Coomassie Blue Staining. (F) Control or OTUD3-overexpressing KYSE180 cells were treated with or without nicotine treatment. Cells were treated with MG132 $(10 \mu \mathrm{M})$ for $6 \mathrm{~h}$ before harvest. Lysates were then immunoprecipitated with anti-ZFP36 antibody, followed by immunoblotting with anti-Ub and anti-ZFP36 antibodies to examine the ubiquitination levels. WCL: whole cell lysate. (G) OTUD3-overexpressing or -silencing KYSE180 cells were treated with MG132 $(10 \mu \mathrm{M})$, and then subjected to ZFP36 ubiquitination analysis. $(H)$ The 293T cells were transfected with HA-Ubwt, HAUbK48, and MYC-OTUD3. Lysates were then subjected to ZFP36 ubiquitination analysis. (I) Analysis of ZFP36 endogenous (left) or K48-linked (right) ubiquitination in KYSE180 cells transfected with FBXW7, or FBXW7 plus OTUD3. (J) Western blot analysis of FBXW7, OTUD3, and ZFP36 in indicated KYSE180 cells. 
A

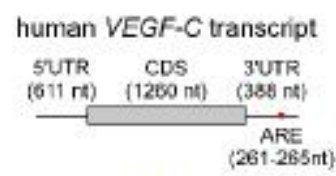

5-tgactatataattatttocactaa-3' Mus.M 5-igactatataantittccatiaa-3" Pan.T 5-1gactatataatttattccactaa-3' Bos. T

5'-tgactatataattatttccactaa-3' Homo.S

5'-tgectatateaeasattcocactee-3' ARE-mut
B

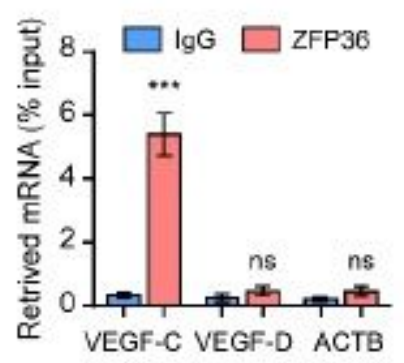

C

Bio-RNA ofry Streptavidin pulldown

D

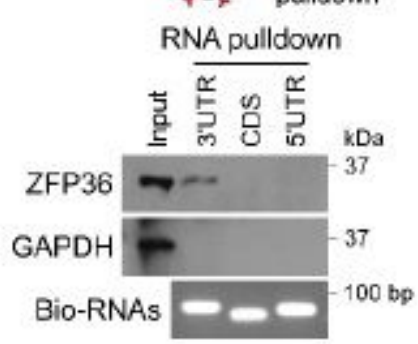

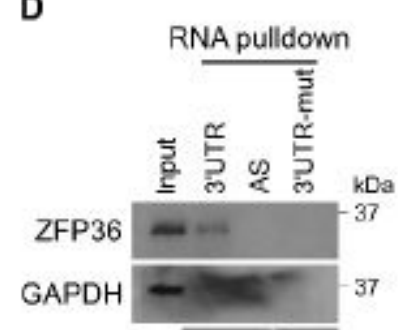

Bio-3'UTR $\longrightarrow-100 \mathrm{bo}$

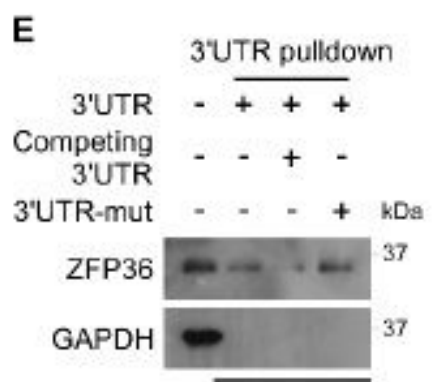

Bio-3'UTR $=-100$ bo
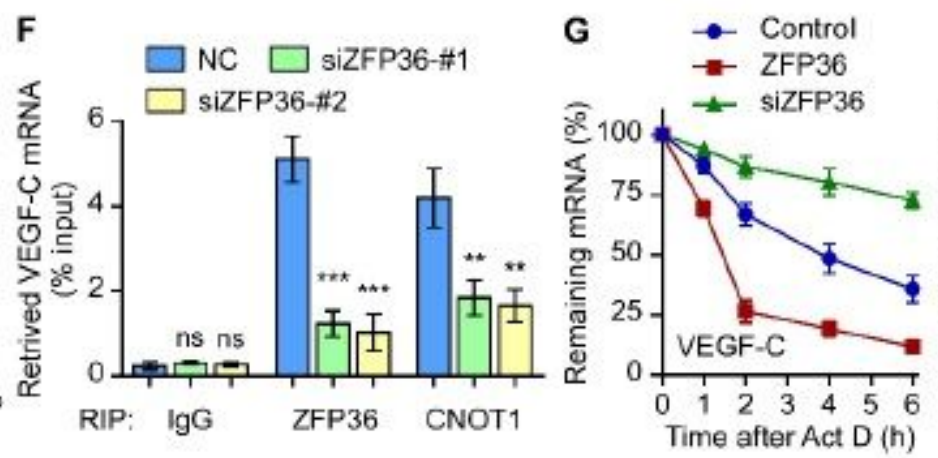

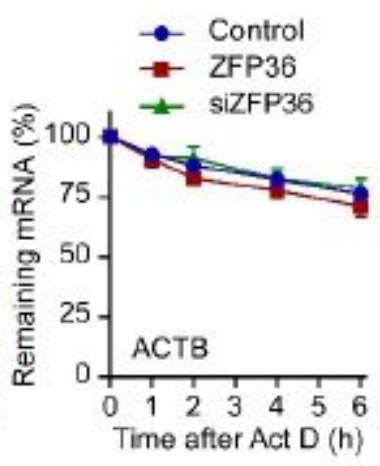

H

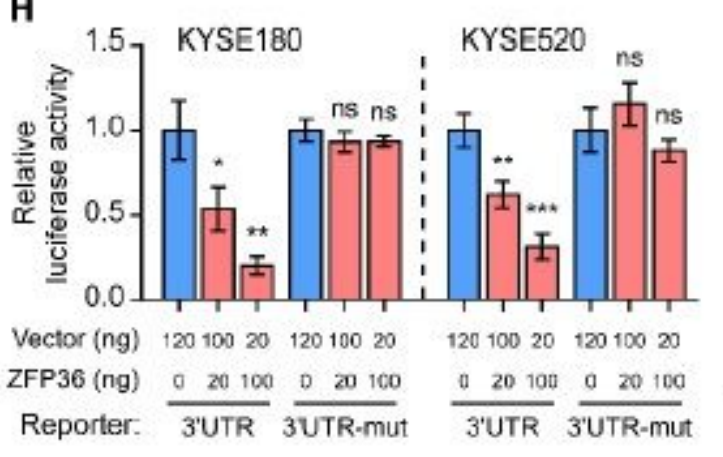

1

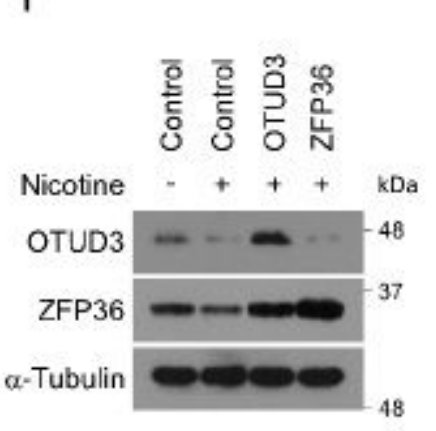

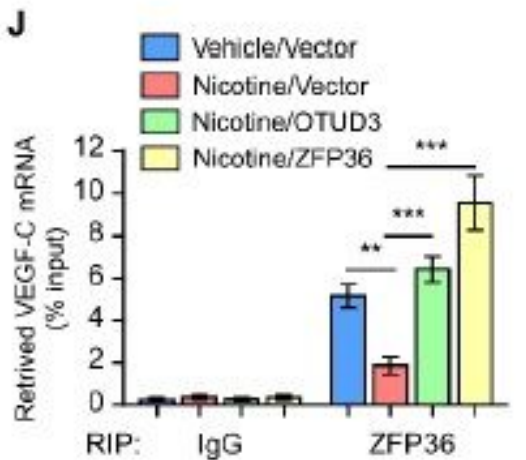

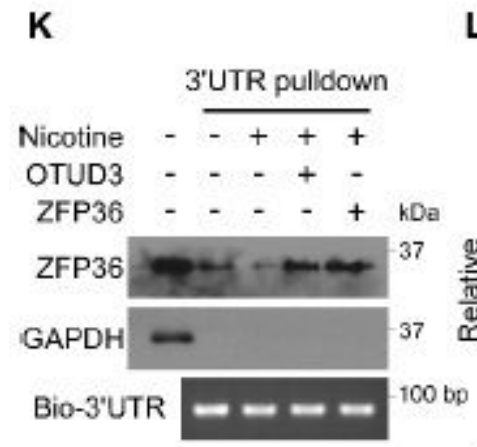

$\mathbf{L}$
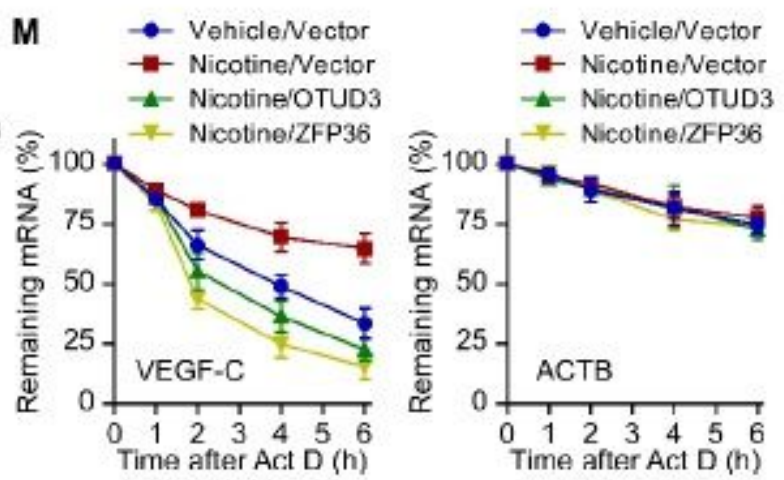

\section{Figure 5}

Nicotine inhibits VEGF-C mRNA decay. (A) The VEGF-C 3'UTR contained a conserved AU-rich element (ARE) among species. (B) RNA immunoprecipitation (RIP) assay was performed in KYSE180 cells using an anti-ZFP36 antibody. Retrieved RNA was then subjected to qRT-PCR analysis of VEGF-C, VEGF-D, and ACTB. IgG was used as a negative control. (C) In vitro-transcribed and biotin-labeled VEGF-C 5'UTR, CDS, and 3'UTR RNAs were incubated with KYSE180 lysates, followed by streptavidin pulldown and detection 
of ZFP36. GAPDH was used as a negative control. (D) Western blot analysis of ZFP36 following the streptavidin pulldown of biotin-labeled VEGF-C 3'UTR, 3'UTR-ARE-mut, or 3'UTR-AS in KYSE180 lysates. (E) Streptavidin pulldown of biotin-labeled VEGF-C 3'UTR with or without competing 3'UTR (unlabeled) or bio-3'UTR-mut. The interaction of ZFP36 protein was examined by western blot. (F) RIP assays of ZFP36 and CNOT1 followed by the qRT-PCR analysis of VEGF-C in control or ZFP36-silencing KYSE180 cells. (G) The half-life of VEGF-C mRNA was traced in indicated KYSE180 cells. ACTB was used as a negative control. (H) Luciferase reporters of VEGF-C 3'UTR and 3'UTR-mut in indicated KYSE180 cells. (I) Western blot analysis of OTUD3 and ZFP36 in KYSE180 cells with or without nicotine treatment. (J) RIP assays of ZFP36 followed by the qRT-PCR analysis of VEGF-C in indicated KYSE180 cells with or without nicotine treatment. (K) Streptavidin pulldown of biotin-labeled VEGF-C 3'UTR in indicated KYSE180 lysates, followed by ZFP36 detection. (L) Luciferase reporters of VEGF-C 3'UTR or 3'UTR-mut in indicated KYSE180 cells. (M) VEGF-C mRNA's half-life was traced in indicated KYSE180 cells with or without nicotine treatment. 

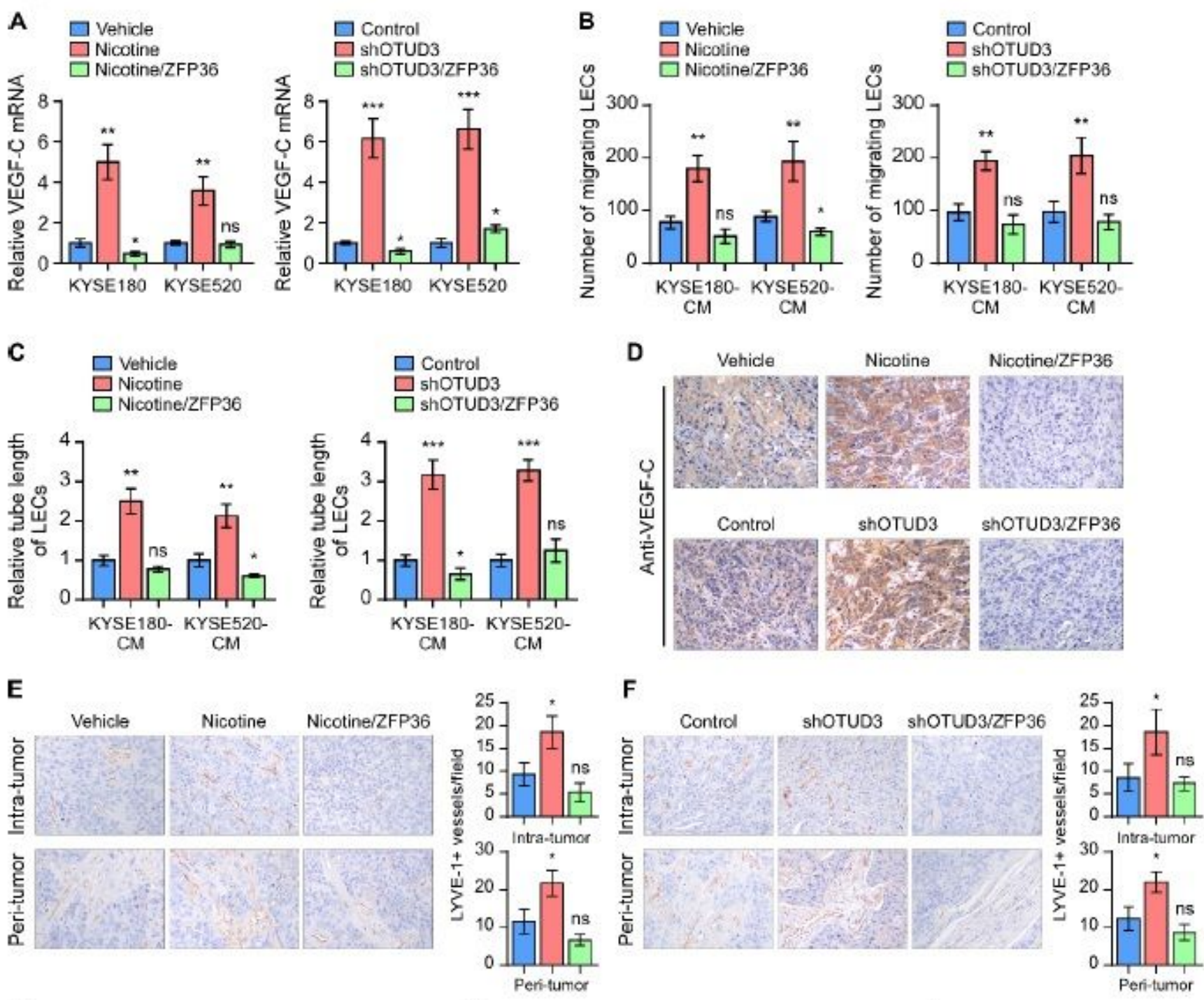

G

\section{H}
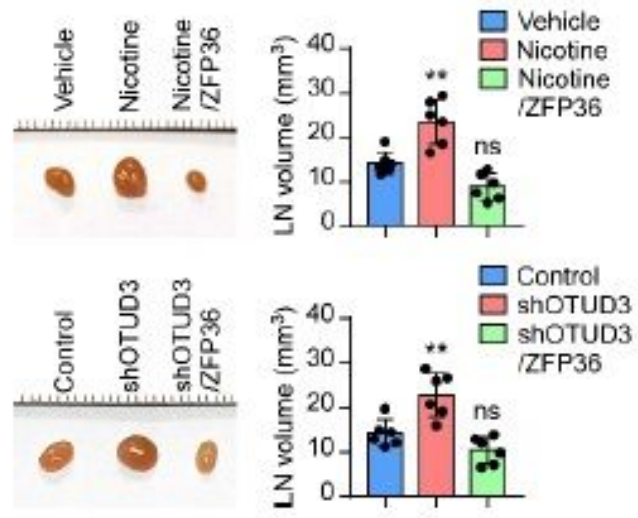
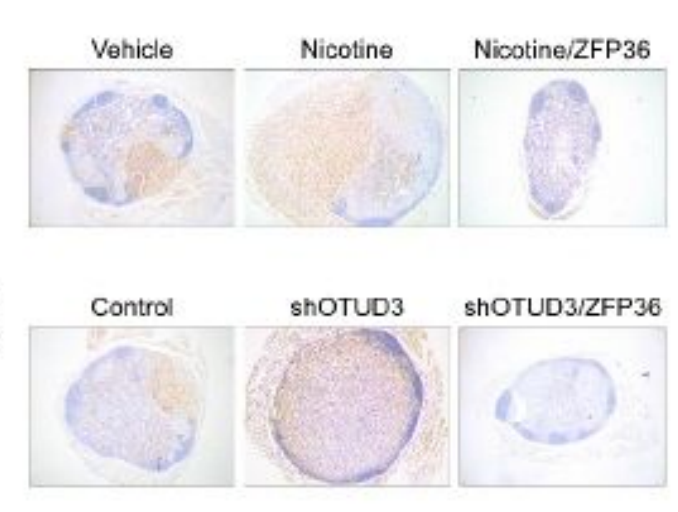
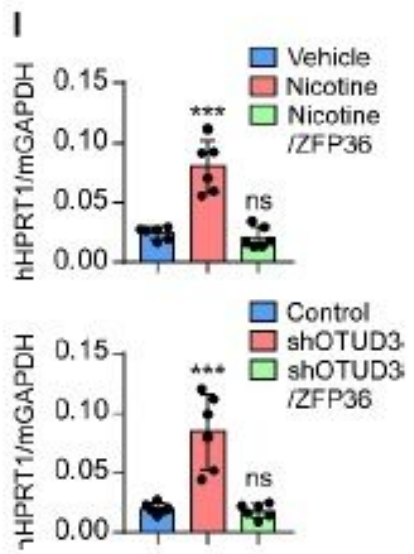

\section{Figure 6}

Induction of VEGF-C mRNA decay abrogates lymphatic metastasis. (A) qRT-PCR analysis of VEGF-C mRNA expression in indicated esophageal cancer cells. (B) Quantification of migrating LECs under treatment of indicated CM. (C) Relative tube length of LECs under treatment of indicated CM. (D) IHC staining of VEGF-C in footpad tumors. (E-F) Representative images of LYVE-1 staining and quantification of intra-tumoral (E) and peri-tumoral (F) lymphatic vessels in indicated footpad tumors. (G) 
Representative image of inguinal LNs. The volumes of LNs from each group were quantified. $(H) I H C$ staining of squamous cell carcinoma marker p63 indicated the metastatic KYSE180 cells in LNs. (I) qRTPCR analysis of human HPRT1 relative to mouse GAPDH in the LNs from each group.
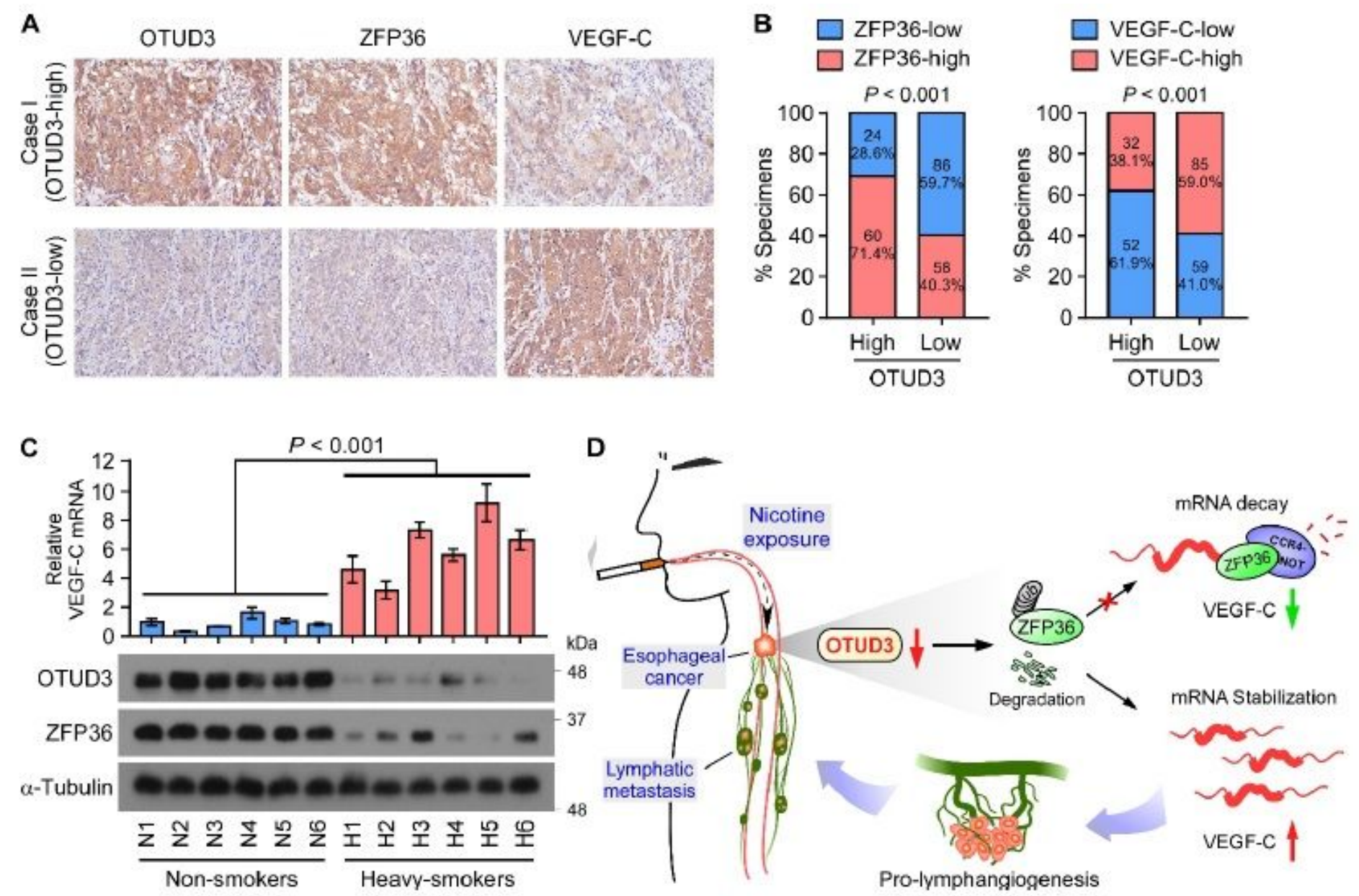

\section{Figure 7}

Clinical relevance and study model. (A) Representative IHC staining images of OTUD3, ZFP36, and VEGF$C$ in esophageal cancer patient specimens $(n=228)$. (B) Correlation analysis showed that OTUD3 was significantly associated with ZFP36 and VEGF-C expression. $\chi 2$ test was used. (C) qRT-PCR of VEGF-C and western blot analysis of OTUD3 and ZFP36 in primary esophageal tumors derived from 6 nonsmoking and 6 heavy-smoking patients. (D) Study model: Nicotine-mediated OTUD3 downregulation facilitates ZFP36 protein degradation and inhibits VEGF-C mRNA decay, leading to robust VEGF-C production, tumor-induced lymphangiogenesis, and lymphatic metastasis in esophageal cancer.

\section{Supplementary Files}

This is a list of supplementary files associated with this preprint. Click to download.

- Supplementaryinformation.pdf 